\title{
Uniqueness for integro-PDE in Hilbert spaces *
}

\author{
ANDRZEJ ŚwięCH \\ School of Mathematics, Georgia Institute of Technology \\ Atlanta, GA 30332, U.S.A. \\ E-mail: swiech@math.gatech.edu \\ AND \\ JERZY ZABCZYK \\ Institute of Mathematics, Polish Academy of Sciences \\ Śniadeckich 8, 00-950 Warsaw, Poland
}

\begin{abstract}
The paper deals with fully nonlinear integro-PDE of Hamilton-Jacobi-Bellman type with unbounded terms in infinite dimensional Hilbert spaces. A notion of viscosity solution is introduced for such integro-PDE and a general comparison theorem is proved.
\end{abstract}

Keywords: viscosity solutions, integro-PDE, Hamilton-Jacobi-Bellman equation, stochastic PDE, Lévy process, Hilbert space.

2010 Mathematics Subject Classification: 49L25, 35R15, 35R09, 49B60, 60G51, $60 \mathrm{H} 15$.

\section{Introduction}

In this paper we begin a systematic study of fully nonlinear integro-PDE in Hilbert spaces, with the goal of the development of a theory of viscosity solutions for such equations. The interest in such equations comes primarily from their connections with infinite dimensional jump-diffusion processes, in particular with stochastic PDE (SPDE) driven by Lévy processes $[8,42,61]$. Integro-PDE may be linear Kolmogorov equations associated with such

*Supported by the MNiSW project N 201419039 "Stochastic equations in infinite dimensional spaces", and NSF grant DMS-0856485. 
SPDE, they may be Bellman-Isaacs equations for stochastic optimal control or stochastic differential game problems driven by SPDE with jumps, they may be infinite dimensional versions of Black-Scholes and Black-Scholes-Barenblatt equations associated with the theory of bond markets driven by jump diffusions. In a recent paper [74], viscosity solutions of integro-PDE were used to prove large deviation results for SPDE with small Lévy noise. There is a need to develop a general theory of such equations. In this manuscript we introduce the notion of viscosity solution appropriate for such equations and deal with the fundamental issue of uniqueness of viscosity solutions. Existence of viscosity solutions and connection with stochastic optimal control problems will be the subject of a future publication.

Linear parabolic PDE with non-local operators on Hilbert spaces have already been a subject of several investigations. The most studied equations are of Kolmogorov's type corresponding to Ornstein-Uhlenbeck processes perturbed by Lévy noise, see e.g. [9, 60]. More general equations with additional first order terms were studied in [49, 64]. In particular [64] contains the so called BEL formula for the gradient of the solutions. Nonlinear parabolic problems with non-local operators, corresponding to optimal stopping problems were investigated in [75] using the theory of maximal monotone operators.

Uniqueness of solutions in the viscosity theory is typically a consequence of a comparison theorem which ensures that under certain conditions a subsolution is always less than or equal to a supersolution. It is usually the more difficult part of the theory. In this paper we prove a general comparison principle for equation (5.1). The proof is rather involved and is divided into several parts. The main difficulty comes from the fact that our integro-PDE has both an integral part and a second order PDE part. If the second order PDE terms are absent, the proof is much easier. A standard proof of comparison for viscosity solutions of second order equations goes through a doubling (of the number of variables) and penalization argument. In finite dimensional spaces one than uses maximum principle for semicontinuous functions (see for instance Theorem 3.2 of [26]) to produce test functions whose second order derivatives have proper ordering. This technique was recently successfully adapted to integro-PDE, see for instance $[12,17,41,62])$. In particular, in [41] a general "non-local maximum principle for semicontinuous functions" was established, which was later generalized in [17]. Unfortunately it is not known if maximum principle for semicontinuous functions is true in infinite dimensions. To remedy this, for a purely second-order PDE, P. L. Lions introduced in [50] a technique which, through a reduction to a finite dimensional case, still allows to produce test functions whose second order derivatives consist of two operators; infinite dimensional ones which are eventually negligible, and finite dimensional ones which have the right ordering. This technique was later slightly improved and generalized in [27] and became a standard tool in the theory 
of second order PDE in Hilbert spaces. Here we combine finite dimensional methods of "non-local maximum principle" [41] with the finite dimensional reduction technique to obtain a kind of "infinite dimensional non-local maximum principle" which is the main tool in the proof of the comparison theorem. We focus our attention on time dependent problems and equations of Bellman type in order to minimize the level of technical details. However, with only minor modifications, the results of this paper can be adapted to stationary equations as well as equations with more general Hamiltonians, for instance to integro-PDE of Isaacs type. Moreover other results typical in the viscosity theory like consistency of viscosity solutions, similar in the spirit to these of [68], can be proved with little effort. We leave these issues to the interested readers.

Finally we mention that the theory of fully nonlinear first and second order integroPDE in finite dimensional spaces is well developed by now. Papers and books [18, 32, $33,34,35,36,43,44,47,48,52,53,54,55,56,57,58,63,69]$ present various PDE, analytic and probabilistic approaches and connections with stochastic optimal control. The theory of viscosity solutions was introduced in [68, 71, 72] and its general theory was further developed in $[2,3,4,11,12,15,16,17,19,23,24,25,37,40,41$, 70]. In particular, papers $[24,70]$ contain higher order regularity results for viscosity solutions. Papers $[13,14]$ treat evolution of interfaces moving with non-local velocity. Papers and books $[1,5,6,7,20,21,39,51,59,62]$ are motivated by applications to finance and control problems.

\section{Preliminaries}

Throughout this paper $H, U$ will be real separable Hilbert spaces equipped with the inner products $\langle\cdot, \cdot\rangle,\langle\cdot, \cdot\rangle_{U}$ and the norms $\|\cdot\|,\|\cdot\|_{U}$, and $A$ will be a linear, densely defined, maximal monotone operator in $H$.

Let $B$ be a bounded, linear, positive, self-adjoint operator on $H$ such that $A^{*} B$ is bounded on $H$ and

$$
\left\langle\left(A^{*} B+c_{0} B\right) x, x\right\rangle \geq 0 \quad \text { for all } x \in H
$$

for some $c_{0} \geq 0$. We refer to $[28,66]$ for existence of such an operator $B$ and various examples. In particular it was shown in [66] that $B=\left((A+I)\left(A^{*}+I\right)\right)^{-1 / 2}$ satisfies $(2.1)$. If $A$ is self-adjoint we can always take $B=(A+I)^{-1}$. Another example of $B$ is given in Example (2.1).

We define for $\gamma>0$ the space $H_{-\gamma}$ to be the completion of $H$ under the norm

$$
\|x\|_{-\gamma}=\left\|B^{\frac{\gamma}{2}} x\right\|
$$


$H_{-\gamma}$ is a Hilbert space equipped with the inner product

$$
\langle x, y\rangle_{-\gamma}=\left\langle B^{\frac{\gamma}{2}} x, B^{\frac{\gamma}{2}} y\right\rangle .
$$

We denote by $\mathcal{L}(H)$ the space of bounded, linear operators on $H$ and by $\mathcal{S}(H)$ the space of bounded, linear, self-adjoint operators in $H$.

Let $\left\{e_{1}, e_{2}, \ldots\right\}$ be an orthonormal basis in $H_{-1}$ made of elements of $H$. For $N \geq 1$ we denote $H_{N}=\operatorname{span}\left\{e_{1}, \ldots, e_{N}\right\}$. We define $P_{N}: H_{-1} \rightarrow H_{-1}$ to be the orthogonal projection onto $H_{N}$ and set $Q_{N}:=I-P_{N}$. We have $P_{N}, Q_{N} \in \mathcal{L}(H)$ and $B P_{N}=P_{N}^{*} B P_{N}, B Q_{N}=$ $Q_{N}^{*} B Q_{N}$. For $x \in H$ we will write $x_{N}=P_{N} x, x_{N}^{\perp}=Q_{N} x$.

We remark that if $B$ is compact then $\left\|B^{\gamma} Q_{N}\right\| \rightarrow 0$ as $N \rightarrow+\infty$ for every $\gamma>0$. Moreover in this case we can take $\left\{e_{1}, e_{2}, \ldots\right\}$ to be a properly normalized orthonormal basis of $H$ composed of eigenvectors of $B$. For such a basis we have $P_{N}=P_{N}^{*}, Q_{N}=Q_{N}^{*}$.

We say that a function $u: \Omega \rightarrow \mathbb{R}$ is $B$-upper-semicontinuous (respectively, $B$ lower-semicontinuous) on $\Omega \subset[0, T] \times H$ if whenever $t_{n} \rightarrow t, x_{n} \rightarrow x, B x_{n} \rightarrow B x$, $(t, x) \in \Omega$, then $\limsup _{n \rightarrow+\infty} u\left(t_{n}, x_{n}\right) \leq u(t, x)$ (respectively, $\liminf _{n \rightarrow+\infty} u\left(t_{n}, x_{n}\right) \geq$ $u(t, x))$. The function $u$ is $B$-continuous on $\Omega$ if it is $B$-upper-semicontinuous and $B$ lower-semicontinuous on $\Omega$.

For an open subset $Z$ of a Hilbert space and an interval $I \subset \mathbb{R}$, we will be using the following function spaces.

$$
\begin{gathered}
B U C(I \times Z)=\{u: I \times Z \rightarrow \mathbb{R}: u \text { is uniformly continuous and bounded }\}, \\
C^{2}(Z)=\left\{u: Z \rightarrow \mathbb{R}: u, D u, D^{2} u \text { are continuous }\right\}, \\
C_{\mathrm{b}}^{2}(Z)=\left\{u \in C^{2}(Z): u \text { is bounded }\right\}, \\
C^{1,2}(I \times Z)=\left\{u: I \times Z \rightarrow \mathbb{R}: u, u_{t}, D u, D^{2} u \text { are continuous }\right\}, \\
C_{\mathrm{b}}^{1,2}(I \times Z)=\left\{u \in C^{1,2}(I \times Z): u \text { is bounded }\right\}, \\
U C^{2}(Z)=\left\{u \in C^{2}(Z): D u, D^{2} u \text { are uniformly continuous }\right\}, \\
U C_{\mathrm{b}}^{2}(Z)=\left\{u \in U C^{2}(Z): u \text { is bounded }\right\}, \\
U C^{1,2}(I \times Z)=\left\{u \in C^{1,2}(I \times Z): u_{t}, D u, D^{2} u \text { are uniformly continuous }\right\}, \\
U C_{\mathrm{b}}^{1,2}(I \times Z)=\left\{u \in U C^{1,2}(I \times Z): u \text { is bounded }\right\},
\end{gathered}
$$

where $D u, D^{2} u$ denote the Fréchet derivatives of $u$ with respect to the space variable.

Let $Y, Z$ be real, separable Hilbert spaces. If $C \in \mathcal{L}(Z)$ is trace class (see e.g. [61], Appendix A.2), its trace is defined by

$$
\operatorname{Tr}(C)=\sum_{i=0}^{\infty}\left\langle C f_{i}, f_{i}\right\rangle_{Z},
$$


where $\left\{f_{1}, f_{2}, \ldots\right\}$ is any orthonormal basis of $Z$. A bounded linear operator $C: Z \rightarrow Y$ is Hilbert-Schmidt if $\sum_{i=0}^{\infty}\left|C f_{i}\right|_{Y}^{2}<+\infty$ for any orthonormal basis of $Z$. The space of Hilbert-Schmidt operators from $Z$ to $Y$ is denoted by $\mathcal{L}_{2}(Z, Y)$. It is equipped with the norm

$$
\|C\|_{\mathcal{L}_{2}(Z, Y)}=\sum_{i=0}^{\infty}\left|C f_{i}\right|_{Y}^{2}=\operatorname{Tr}\left(C^{*} C\right)
$$

which is independent of the choice of basis. Moreover $\|C\|_{\mathcal{L}_{2}(Z, Y)}=\left\|C^{*}\right\|_{\mathcal{L}_{2}(Y, Z)}$.

Example 2.1. The setting below is needed to study a controlled hyperbolic system with Lévy noise (see [74], Section 9) considered in Example 4.3.

Let $A$ be a strictly positive, self-adjoint operator in $H$ with a bounded inverse. Then the operator

$$
\mathcal{A}=\left(\begin{array}{cc}
0 & -I \\
A & 0
\end{array}\right), \quad \mathcal{D}(\mathcal{A})=\left(\begin{array}{c}
D(A) \\
\times \\
D\left(A^{1 / 2}\right)
\end{array}\right)
$$

is maximal monotone in the Hilbert space $\mathcal{H}=\left(\begin{array}{c}D\left(A^{1 / 2}\right) \\ \times \\ H\end{array}\right)$, equipped with the inner product

$$
\left\langle\left(\begin{array}{l}
u \\
v
\end{array}\right),\left(\begin{array}{l}
\bar{u} \\
\bar{v}
\end{array}\right)\right\rangle_{\mathcal{H}}=\left\langle A^{1 / 2} u, A^{1 / 2} \bar{u}\right\rangle_{H}+\langle v, \bar{v}\rangle_{H}, \quad\left(\begin{array}{l}
u \\
v
\end{array}\right),\left(\begin{array}{l}
\bar{u} \\
\bar{v}
\end{array}\right) \in \mathcal{H} .
$$

Moreover, $\mathcal{A}^{*}=-\mathcal{A}$ and one easily checks that the operator

$$
\mathcal{B}=\left(\begin{array}{cc}
A^{-1 / 2} & 0 \\
0 & A^{-1 / 2}
\end{array}\right)
$$

is bounded, positive, self-adjoint on $\mathcal{H}, \mathcal{A}^{*} \mathcal{B}$ is bounded, and (2.1) holds with any constant $c_{0} \geq 0$. Moreover

$$
\left\|\left(\begin{array}{l}
u \\
v
\end{array}\right)\right\|_{-1}=\left(\left\|A^{1 / 4} u\right\|^{2}+\left\|A^{-1 / 4} v\right\|^{2}\right)^{1 / 2}, \quad\left(\begin{array}{l}
u \\
v
\end{array}\right) \in \mathcal{H} .
$$

\section{Optimal control problem and HJB equation}

Let $L$ be a Lévy process on $[0,+\infty)$ in $U$. Then

$$
\mathbb{E} e^{i\langle\lambda, L(t)\rangle_{U}}=e^{-t \psi(\lambda)},
$$

where for $\lambda \in U$,

$$
\psi(\lambda)=-i\langle m, \lambda\rangle_{U}+\frac{1}{2}\langle Q \lambda, \lambda\rangle_{U}+\int_{U \backslash\{0\}}\left(1-e^{i\langle\lambda, y\rangle_{U}}+\mathbf{1}_{\left\{\|y\|_{U}<1\right\}} i\langle\lambda, y\rangle_{U}\right) \nu(d y),
$$


$m \in U, Q \geq 0$ is a bounded, self-adjoint operator on $U$ such that $\operatorname{Tr}(Q)<+\infty$, and $\nu$ is a non-negative measure on $(U \backslash\{0\}, \mathcal{B}(U \backslash\{0\}))$, where $\mathcal{B}(U \backslash\{0\})$ is the Borel $\sigma$-field, for which

$$
\int_{0<\|y\|_{U}<1}\|y\|_{U}^{2} \nu(d y)+\int_{\|y\|_{U} \geq 1} \nu(d y)<+\infty .
$$

The measure $\nu$ is called the Lévy measure of $L$ or the jump intensity measure of $L$ and the function $\psi$, the Lévy exponent of $L$ or the characteristic exponent of $L$. According to formula (3.1), the process $L$ can be represented in the form

$$
L(t)=m t+W(t)+L_{0}(t)+L_{1}(t)
$$

where $W, L_{0}, L_{1}$ are independent Lévy processes such that $W$ is a $Q$-Wiener process in $U$ (see [30]), and

$$
L_{0}(t)=\int_{0}^{t} \int_{0<\|y\|_{U}<1} y \hat{\pi}(d s, d y), \quad L_{1}(t)=\int_{0}^{t} \int_{\|y\|_{U} \geq 1} y \pi(d s, d y),
$$

where $\pi$ is the Poisson random measure of jumps of $L$ and $\hat{\pi}$ is the compensated Poisson random measure of jumps:

$$
\begin{gathered}
\left.\pi([0, t], B)=\sum_{0<s \leq t} \mathbf{1}_{B}(L(s)-L(s-)), \quad B \in \mathcal{B}(U \backslash\{0\})\right), L(s-)=\lim _{t \uparrow s} L(t), \\
\hat{\pi}(d t, d y)=\pi(d t, d y)-d t \nu(d y)
\end{gathered}
$$

(see e.g. $[10,22,61,67]$ ). The process $L_{0}$ is a square integrable martingale, and $L_{1}$ is a compound Poisson process. In particular, for arbitrary trace class, positive definite operator $R$ on $U$ and $\alpha \in(0,2)$, the following function

$$
\psi(\lambda)=\langle R \lambda, \lambda\rangle_{U}^{\frac{\alpha}{2}}, \quad \lambda \in U
$$

is of the form (3.1). It corresponds to an $\alpha$-stable Lévy process with the Lévy measure

$$
\nu=\frac{\Gamma(1-\alpha)}{\alpha} \int_{0}^{+\infty} N(0, s R) \frac{1}{s^{1+\alpha}} d s,
$$

where $N(0, s R)$ is the Gaussian measure on $U$ with mean 0 and the covariance $s R$.

Let $T>0$. We consider a family of the following stochastic optimal control problems. For a given $t \in[0, T]$, the set of admissible controls, denoted by $\mathcal{U}_{t}$, will be the collection of all 6-tuples $\left(\Omega, \mathcal{F}, \mathcal{F}_{s}^{t}, \mathbb{P},(W, L), a(\cdot)\right)$ satisfying the following conditions:

(i) $(\Omega, \mathcal{F}, \mathbb{P})$ is a complete probability space; 
(ii) $(W, L)=\{(W(s), L(s)): t \leq s \leq T\}$ is a pair of an independent $U$-valued $Q$ Wiener process $W(s)(Q \in \mathcal{S}(U), Q \geq 0$ and $Q$ is trace-class), and a $U$-valued Lévy process $L(s)=L_{0}(s)+L_{1}(s)$ as above defined on $(\Omega, \mathcal{F}, \mathbb{P})$ beginning at $t$ (with $W(t)=L_{0}(t)=L_{1}(t)=0, \mathbb{P}$ a.s. $)$, and $\mathcal{F}_{s}^{t}$ is the filtration generated by $(W, L)$, augmented by the $\mathbb{P}$-null sets in $\mathcal{F}$;

(iii) $a:[t, T] \times \Omega \rightarrow \Gamma$, is an $\mathcal{F}_{s}^{t}$-adapted, cádlág (right continuous with left limits) processes with values in a complete separable metric space $\Gamma$.

Consider a family of abstract stochastic differential equations (SDE)

$$
\left\{\begin{array}{l}
d X(s)=(-A X(s)+b(X(s), a(s))) d s+\sigma(X(s), a(s)) d W(s) \\
+\int_{0<\|y\|_{U}<1} \gamma(X(s-), a(s-), y) \hat{\pi}(d s, d y)+\int_{\|y\|_{U} \geq 1} \gamma(X(s-), a(s-), y) \pi(d s, d y) \\
X(t)=x \in H
\end{array}\right.
$$

The above SDE is quite general and it includes for instance stochastic semilinear parabolic and hyperbolic problems with Lévy noise [74] to name a few. In particular, if $\gamma$ is a linear transformation with respect to the third variable, i.e.

$$
\gamma(x, a, y)=\bar{\gamma}(x, a) y
$$

we arrive at the equation

$$
d X(s)=(-A X(s)+b(X(s), a(s))) d s+\sigma(X(s), a(s)) d W(s)+\bar{\gamma}(X(s-), a(s-) d L(s) .
$$

We want to minimize a cost functional of the form

$$
J(t, x ; a(\cdot))=\mathbb{E}\left\{\int_{t}^{T} f(X(s), a(s)) d s+g(X(T))\right\}
$$

over all admissible controls in $\mathcal{U}_{t}$. The dynamic programming equation for this problem is an integro-PDE Hamilton-Jacobi-Bellman (HJB) equation

$$
\left\{\begin{array}{l}
u_{t}-\langle A x, D u\rangle+\inf _{a \in \Gamma}\left\{\frac{1}{2} \operatorname{Tr}\left(\left(\sigma(x, a) Q^{\frac{1}{2}}\right)\left(\sigma(x, a) Q^{\frac{1}{2}}\right)^{*} D^{2} u\right)+\langle b(x, a), D u\rangle+f(x, a)\right. \\
\left.+\int_{U \backslash\{0\}}\left(u(t, x+\gamma(x, a, y))-u(t, x)-\mathbf{1}_{\left\{\|y\|_{U}<1\right\}}\langle\gamma(x, a, y), D u(t, x)\rangle\right) \nu(d y)\right\}, \\
u(T, x)=g(x) .
\end{array}\right.
$$

The above integro-PDE should be satisfied by the value function

$$
V(t, x)=\inf _{a(\cdot) \in \mathcal{U}_{t}} J(t, x ; a(\cdot)) .
$$

The validity of this is the subject of a paper under preparation. 


\section{Assumptions}

For $(x, p, X, v) \in H \times H \times \mathcal{S}(H) \times U C_{\mathrm{b}}^{2}(H)$, we set

$$
\begin{aligned}
& F(x, p, X, v)=\inf _{a \in \Gamma}\left\{\frac{1}{2} \operatorname{Tr}\left(\sigma(x, a) \sigma^{*}(x, a) X\right)+\langle b(x, a), p\rangle+f(x, a)\right. \\
& \left.\quad+\int_{U \backslash\{0\}}\left(v(x+\gamma(x, a, y))-v(x)-\langle\gamma(x, a, y), D v(x)\rangle \mathbf{1}_{\left\{\|y\|_{U}<1\right\}}\right) \nu(d y)\right\}
\end{aligned}
$$

(Above $\sigma$ corresponds to $\sigma(x, a) Q^{\frac{1}{2}}$ in (3.5).)

We will be making the following assumptions. Some of them can be relaxed but we do not state them in the most general form in order not to overcomplicate the paper which is already very technical.

(i) There exists a Borel measurable function $\rho$, bounded on bounded sets, such that $\inf _{\{\|z\|>r\}} \rho(z)>0$ for every $r>0$, and

$$
\int_{H}\left[(\rho(z))^{2} \mathbf{1}_{\left\{\|z\|_{U}<1\right\}}+\mathbf{1}_{\left\{\|z\|_{U} \geq 1\right\}}\right] \nu(d z)<+\infty .
$$

(ii) $\sigma: H \times \Gamma \rightarrow \mathcal{L}_{2}(U, H), b: H \times \Gamma \rightarrow H$ are continuous and such that

$$
\|b(x, a)-b(y, a)\|,\|\sigma(x, a)-\sigma(y, a)\|_{\mathcal{L}_{2}(U, H)} \leq C\|x-y\|_{-1},
$$

$\gamma: H \times \Gamma \times U \rightarrow H$ is continuous in $x, a$, Borel measurable with respect to $z$ and such that

$$
\|\gamma(x, a, z)-\gamma(y, a, z)\| \leq C \rho(z)\|x-y\|_{-1}
$$

for all $x, y \in H, z \in U, a \in \Gamma$, where $\Gamma$ is a complete separable metric space.

(iii) $f: H \times \Gamma \rightarrow \mathbb{R}, g: H \rightarrow \mathbb{R}$ are continuous and such that

$$
|f(x, a)-f(y, a)|+|g(x)-g(y)| \leq \omega\left(\|x-y\|_{-1}\right)
$$

for all $x, y \in H, a \in \Gamma$, where $\omega$ is a modulus of continuity.

(iv)

$$
\begin{gathered}
\|\sigma(0, a)\|_{\mathcal{L}_{2}(U, H)},\|b(0, a)\|,|f(0, a)| \leq C, \\
\|\gamma(0, a, z)\| \leq C \rho(z)
\end{gathered}
$$

for all $a \in \Gamma, z \in U$.

$(\mathrm{v})$

For every $x \in H, z \in U$, the set $\{\gamma(x, a, z): a \in \Gamma\}$ is precompact in $H$. 
(vi)

$$
\lim _{N \rightarrow+\infty} \sup _{a \in \Gamma}\left|\operatorname{Tr}\left(\sigma(x, a) \sigma^{*}(x, a) B Q_{N}\right)\right|=0
$$

for every $x \in H$.

We notice that under the above assumptions, the Hamiltonian $F$ in (4.1) is well defined. We remark that for $\gamma$ as in (3.4) we have $\rho(z)=\|z\|_{U}$.

Remark 4.1. It is easy to see that (4.8) implies that

$$
\lim _{N \rightarrow+\infty} \sup _{a \in \Gamma}\left\|Q_{N} \gamma(x, a, z)\right\|_{-1}=0
$$

for every $x \in H, z \in U$. Moreover, (4.8) holds if $\Gamma$ is compact.

Remark 4.2. It is not difficult to notice (see for instance [45]) that if (4.3) and (4.6) hold, then (4.9) is satisfied if $B$ is compact, or if the control set $\Gamma$ is compact. Moreover, $\operatorname{Tr}\left(\sigma(x, a) \sigma^{*}(x, a) B Q_{N}\right) \geq 0$ for every $x \in H, a \in \Gamma$.

Example 4.3. (Controlled wave equation with Lévy noise.) Consider the equation

$$
\left\{\begin{array}{lc}
\frac{\partial^{2} u}{\partial s^{2}}(s, \xi)=\Delta u(s, \xi)+h(\xi, u(s, \xi), a(s))+<k(\xi, u(s-, \xi), a(s-)), \frac{\partial}{\partial s} L(s)>_{U}, & s>t, \xi \in \mathcal{O}, \\
u(s, \xi)=0, & \xi \in \mathcal{O}, \\
u(t, \xi)=u_{0}(\xi), & \xi \in \mathcal{O},
\end{array}\right.
$$

where $\mathcal{O}$ a bounded regular domain in $\mathbb{R}^{d}, u_{0} \in H_{0}^{1}(\mathcal{O}), v_{0} \in L^{2}(\mathcal{O})$, and $L$ is an $\alpha$-stable process in a Hilbert space $U$ with the Lévy exponent (3.2). In addition $h, k$ are mappings from $\mathcal{O} \times R^{1} \times \Gamma$ into respectively $R^{1}$ and $U$. In this case we take $H=L^{2}(\mathcal{O})$ and $A=\Delta$ with the domain $D(A)=H^{2}(\mathcal{O}) \cap H_{0}^{1}(\mathcal{O})$. Then $D\left(A^{\frac{1}{2}}\right)=H_{0}^{1}(\mathcal{O})$ and

$$
\mathcal{H}=\left(\begin{array}{c}
H_{0}^{1}(\mathcal{O}) \\
\times \\
L^{2}(\mathcal{O})
\end{array}\right)
$$

Moreover

$$
\mathcal{A}=\left(\begin{array}{cc}
0 & -I \\
-\Delta & 0
\end{array}\right), \quad \mathcal{D}(\mathcal{A})=\left(\begin{array}{c}
H^{2}(\mathcal{O}) \cap H_{0}^{1}(\mathcal{O}) \\
\times \\
H_{0}^{1}(\mathcal{O})
\end{array}\right)
$$

Equation (4.11) can be rewritten as an evolution equation

$$
d X(s)=(-\mathcal{A} X(s)+b(X(s), a(s))) d t+\bar{\gamma}(X(s-), a(s-)) d L(s), \quad X(t)=x=\left(\begin{array}{l}
u_{0} \\
v_{0}
\end{array}\right)
$$


where

$$
b\left(\left(\begin{array}{l}
u \\
v
\end{array}\right), a\right)=\left(\begin{array}{c}
0 \\
h(\cdot, u(\cdot), a)
\end{array}\right), \quad \bar{\gamma}\left(\left(\begin{array}{l}
u \\
v
\end{array}\right), a\right) y=\left(\begin{array}{c}
0 \\
<k(\cdot, u(\cdot), a), y>_{U}
\end{array}\right) .
$$

Note that (4.2) is satisfied if $\rho(z)=\|z\|_{U}$. Assume in addition that $h, k$ are continuous in all variables and $h(\xi, \cdot, a), k(\xi, \cdot, a)$ are Lipschitz continuous. Denote by $c_{h}(\xi, a)$ and $c_{h}(\xi, a)$ their Lipschitz constants. We show that if

$$
c_{h}=\sup _{\xi, a} c_{h}(\xi, a)<+\infty, \quad c_{k}=\sup _{\xi, a} c_{k}(\xi, a)<+\infty
$$

then the crucial assumption (ii) is satisfied. Indeed

$$
\begin{aligned}
& \left\|b\left(\left(\begin{array}{l}
u \\
v
\end{array}\right), a\right)-b\left(\left(\begin{array}{c}
\tilde{u} \\
\tilde{v}
\end{array}\right), a\right)\right\|_{\mathcal{H}}=\|h(\cdot, u(\cdot), a)-h(\cdot, \tilde{u}(\cdot), a)\|_{L^{2}(\mathcal{O})} \\
& =\left(\int_{\mathcal{O}}|h(\xi, u(\xi), a)-h(\xi, \tilde{u}(\xi), a)|^{2} d \xi\right)^{\frac{1}{2}} \leq c_{h}\left(\int_{\mathcal{O}}|u(\xi)-\tilde{u}(\xi)|^{2} d \xi\right)^{\frac{1}{2}}=c_{h}\|u-\tilde{u}\|_{H} \\
& \leq c\left\|A^{1 / 4}(u-\tilde{u})\right\|_{H} \leq c\left\|\left(\begin{array}{c}
u \\
v
\end{array}\right)-\left(\begin{array}{c}
\tilde{u} \\
\tilde{v}
\end{array}\right)\right\|_{-1}
\end{aligned}
$$

for some constant c, where we used Example 2.1 to obtain the last line above. Similar calculations show that that (4.4) is satisfied as well.

\section{$5 \quad$ Viscosity solutions}

We consider second order terminal value problems for integro-PDE of the form

$$
\left\{\begin{array}{l}
u_{t}-\langle A x, D u\rangle+F\left(x, u, D u, D^{2} u, u(t, \cdot)\right)=0, \quad \text { in }(0, T) \times H, \\
u(T, x)=g(x),
\end{array}\right.
$$

where $F$ is defined by (4.1). The definition of viscosity solution depends on the domain of $F$, which is determined by the integrability condition we impose on the measure $\nu(d z)$. In this paper we assume (4.2), i.e. we focus on bounded viscosity solutions. Our definition is based on the notion of the so called $B$-continuous viscosity solution which was introduced for first order equations in $[28,29]$. The reader should not consider our choice of test functions as a definitive one but rather as a choice which gives good theory and which can be modified if there is a need to do so.

Definition 5.1. Let (4.2) be satisfied. We will say that a function $\psi$ is a test function if $\psi=\varphi+\delta(t, x) h(\|x\|)$, where:

(i) $\varphi, \delta \in U C_{\mathrm{b}}^{1,2}((\epsilon, T-\epsilon) \times H), \delta \geq 0, \varphi$ is B-lower-semicontinuous, $\delta$ is B-continuous, and $A^{*} D \varphi, A^{*} D \delta$ are uniformly continuous on $(\epsilon, T-\epsilon) \times H$ for every $\epsilon>0$. 
(ii) $h \in U C_{\mathrm{b}}^{2}(\mathbb{R}), h$ is even, $h^{\prime}(r) \geq 0$ for $r \in(0,+\infty)$.

Definition 5.2. Let (4.2) be satisfied. A bounded B-upper semicontinuous function u: $(0, T) \times H \rightarrow \mathbb{R}$ is a viscosity subsolution of (5.1) if whenever $u-\psi$ has a global maximum at a point $(t, x)$ for a test function $\psi(s, y)=\varphi(s, y)+\delta(s, y) h(\|y\|)$ then

$$
\begin{gathered}
\psi_{t}(t, x)-\left\langle x, A^{*} D \varphi(t, x)+h(\|x\|) A^{*} D \delta(t, x)\right\rangle \\
+F\left(x, D \psi(t, x), D^{2} \psi(t, x), \psi(t, \cdot)\right) \geq 0 .
\end{gathered}
$$

$A$ bounded B-lower semicontinuous function $u:(0, T) \times H \rightarrow \mathbb{R}$ is a viscosity supersolution of (5.1) if whenever $u+\psi$ has a global minimum at a point $(t, x)$ for a test function $\psi$ then

$$
\begin{aligned}
& -\psi_{t}(t, x)+\left\langle x, A^{*} D \varphi(t, x)+h(\|x\|) A^{*} D \delta(t, x)\right\rangle \\
& \quad+F\left(x,-D \psi(t, x),-D^{2} \psi(t, x),-\psi(t, \cdot)\right) \leq 0 .
\end{aligned}
$$

A viscosity solution of (5.1) is a function which is both a viscosity subsolution and a viscosity supersolution.

Remark 5.3. We do not need it in this paper but sometimes it may be useful to extend the class of test functions to be $\psi(s, y)=\varphi(s, y)+\sum_{i=1}^{n} \delta_{i}(s, y) h_{i}(\|y\|)$, where $\varphi, \delta_{i}, h_{i}$ are as in Definition 5.1 .

We will need a "localized" definition of viscosity solution which will allow us to use test functions which are not necessarily bounded. Localized definitions of viscosity solutions first appeared in $[72,68]$.

For $0<r<1,(x, p, X, v, w) \in H \times H \times \mathcal{S}(H) \times U C^{2}(H) \times B U C(H)$, we set

$$
\begin{aligned}
F_{r}(x, p, X, v, w) & =\inf _{a \in \Gamma}\left\{\frac{1}{2} \operatorname{Tr}\left(\sigma(x, a) \sigma^{*}(x, a) X\right)+\langle b(x, a), p\rangle+f(x, a)\right. \\
& +\int_{\left\{0<\|y\|_{U}<r\right\}}(v(x+\gamma(x, a, y))-v(x)-\langle\gamma(x, a, y), D v(x)\rangle) \nu(d y) \quad(5.6) \\
& \left.+\int_{\left\{\|y\|_{U} \geq r\right\}}\left(w(x+\gamma(x, a, y))-w(x)-\langle\gamma(x, a, y), p\rangle \mathbf{1}_{\left\{\|y\|_{U}<1\right\}}\right) \nu(d y)\right\} .
\end{aligned}
$$

Definition 5.4. Let (4.2) be satisfied. We will say that a function $\psi$ is a test function in the sense of Definition 5.4 if $\psi=\varphi+h(\|x\|)$, where $\varphi$, $h$ are as in Definition 5.1 without being bounded, however $\varphi$ must be bounded on every set $(\epsilon, T-\epsilon) \times\left\{x:\|x\|_{-1} \leq R\right\}$, $0<\epsilon<T, R>0$.

$A$ bounded $B$-upper semicontinuous function $u:(0, T) \times H \rightarrow \mathbb{R}$ is a viscosity subsolution of (5.1) in the sense of Definition 5.4 if whenever $u-\psi$ has a global maximum at a point $(t, x)$ for a test function $\psi(s, y)=\varphi(s, y)+h(\|y\|)$ then for every $r>0$

$$
\psi_{t}(t, x)+\left\langle x, A^{*} D \varphi(t, x)\right\rangle+F_{r}\left(x, D \psi(t, x), D^{2} \psi(t, x), \psi(t, \cdot), u(t, \cdot)\right) \geq 0 .
$$


$A$ bounded B-lower semicontinuous function $u:(0, T) \times H \rightarrow \mathbb{R}$ is a viscosity supersolution of (5.1) in the sense of Definition 5.4 if whenever $u+\psi$ has a global minimum at a point $(t, x)$ for a test function $\psi=\varphi(s, y)+h(\|y\|)$ then for every $r>0$

$$
-\psi_{t}(t, x)-\left\langle x, A^{*} D \varphi(t, x)\right\rangle+F_{r}\left(x,-D \psi(t, x),-D^{2} \psi(t, x),-\psi(t, \cdot), u(t, \cdot)\right) \leq 0 .
$$

A viscosity solution of (5.1) is a function which is both a viscosity subsolution and a viscosity supersolution.

The additional requirement that the test function $\varphi$ be bounded on every set $(\epsilon, T-$ $\epsilon) \times\left\{\|x\|_{-1} \leq R\right\}$ is needed to guarantee that without loss of generality we can always assume that the test functions $\varphi$ and $h(\|y\|)$ in the definition of viscosity solution are bounded. We refer to Remark 4.3 of [73] for a simple construction on how to achieve this.

We assume in Lemma 5.5 (and similarly in Theorem 6.2) that the functions involved are in $B U C\left((0, T) \times H_{-1}\right)$ for simplicity. We only need that they are defined on $(0, T) \times H$ and are uniformly continuous in the $|\cdot| \times\|\cdot\|_{-1}$ norm. However then they can be extended naturally to functions in $B U C\left((0, T) \times H_{-1}\right)$.

Lemma 5.5. Let (4.2)-(4.8) be satisfied. If a function $u \in B U C\left((0, T) \times H_{-1}\right)$ is a viscosity subsolution (respectively, supersolution) of (5.1) then it is a viscosity subsolution (respectively, supersolution) of (5.1) in the sense of Definition 5.4.

Proof. We notice that, by Remark 4.1, (4.10) is satisfied. Let $u \in B U C\left((0, T) \times H_{-1}\right)$ be a viscosity subsolution of (5.1) in the sense of Definition 5.2, and let $u-\psi$ has a global maximum at point $(t, x)$ for a test function $\psi(s, y)=\varphi(s, y)+h(\|y\|)$, where without loss of generality we can assume that $\varphi$ and $h(\|y\|)$ are bounded, and $u(t, x)=\psi(t, x)$. Let $r>0$. Since $u \in B U C\left((0, T) \times H_{-1}\right)$, there exists a modulus $\sigma$ such that

$$
|u(s, y)-u(s, z)| \leq \sigma\left(\|y-z\|_{-1}\right) \quad \text { for } s \in(0, T), y, z \in H
$$

We can assume that $\sigma(\tau) \leq 2\|u\|_{\infty}=M, \tau \geq 0$. It follows from (4.4) and (4.7) that $\|\gamma(x, a, y)\| \leq C(x) \rho(y)$. Set $c:=C(x)$ and let for $\epsilon>0$

$$
k_{\epsilon}=\int_{\left\{\rho(y) \geq \frac{\epsilon}{c}\right\} \cup\left\{\|y\|_{U} \geq r\right\}} \nu(d y)
$$

and

$$
\delta_{\epsilon}=\frac{\epsilon}{4\left(1+k_{\epsilon}\right)}
$$

For $N \geq 1$ let $u_{N}^{\epsilon}$ be a smooth approximation of $u_{\mid H_{N}}$ such that

$$
u_{\mid H_{N}} \leq u_{N}^{\epsilon} \leq u_{\mid H_{N}}+\delta_{\epsilon}
$$


Set

$$
\tilde{u}_{N}^{\epsilon}(s, y)=u_{N}^{\epsilon}\left(s, P_{N} y\right)+2 \delta_{\epsilon}+h_{\epsilon}\left(\left\|Q_{N} y\right\|_{-1}\right),
$$

where $h_{\epsilon}$ is a smooth bounded, non-decreasing function such that $h_{\epsilon}(\tau) \leq C_{\epsilon} \tau^{2}$ for $\tau \geq$ $0, h_{\epsilon}(\tau)=2 M+1$ for $\tau \geq 1$, and $\sigma(\tau) \leq \delta_{\epsilon}+h_{\epsilon}(\tau)$. Then $u \leq \tilde{u}_{N}^{\epsilon}$ and

$$
\tilde{u}_{N}^{\epsilon}(s, y) \leq u(s, y)+2\left(2 \delta_{\epsilon}+h_{\epsilon}\left(\left\|Q_{N} y\right\|_{-1}\right)\right) \leq \psi(s, y)+2\left(2 \delta_{\epsilon}+h_{\epsilon}\left(\left\|Q_{N} y\right\|_{-1}\right)\right),
$$

which in particular imply that

$$
\left|u(s, y)-\tilde{u}_{N}^{\epsilon}(s, y)\right| \leq 2\left(2 \delta_{\epsilon}+h_{\epsilon}\left(\left\|Q_{N} y\right\|_{-1}\right)\right) .
$$

Let $\eta:[0, \infty) \rightarrow[0,1]$ be a smooth function such that $\eta(\tau)=1$ for $\tau<1, \eta(\tau)=0$ for $\tau>2$, and which is strictly decreasing on $[1,2]$. We define $\eta_{\epsilon}(\tau)=\eta(\tau / \epsilon)$ and

$$
\tilde{\psi}_{N}^{\epsilon}(s, y)=\psi(s, y) \eta_{\epsilon}\left(\|y-x\|_{-1}\right)+\tilde{u}_{N}^{\epsilon}(s, y)\left(1-\eta_{\epsilon}\left(\|y-x\|_{-1}\right)\right) .
$$

We notice that by (5.7) and the definition of $c$,

$$
\begin{gathered}
\tilde{\psi}_{N}^{\epsilon}(t, x+\gamma(x, a, y))=\psi(t, x+\gamma(x, a, y)) \quad \text { if } \rho(y) \leq \epsilon / c, a \in \Gamma \\
\tilde{\psi}_{N}^{\epsilon}(t, y) \leq \psi(t, y)+2\left(2 \delta_{\epsilon}+h_{\epsilon}\left(\left\|Q_{N} y\right\|_{-1}\right)\right)
\end{gathered}
$$

It is now clear that $u-\tilde{\psi}_{N}^{\epsilon}$ has a global maximum at $(t, x)$. Therefore, by Definition 5.2 , we have

$$
\begin{aligned}
0 \leq \psi_{t}(t, x)+\left\langle x, A^{*} D \varphi(t, x)\right\rangle+ & \inf _{a \in \Gamma}\left\{\frac{1}{2} \operatorname{Tr}\left(\sigma(x, a) \sigma^{*}(x, a) D^{2} \psi(t, x)\right)\right. \\
& +\langle b(x, a), D \psi(t, x)\rangle+f(x, a) \\
+ & \left.\left.\int_{U \backslash\{0\}}\left(\tilde{\psi}_{N}^{\epsilon}(t, x+\gamma(x, a, y))-\tilde{\psi}_{N}^{\epsilon}(t, x)-\langle\gamma(x, a, y), D \psi(t, x)\rangle \mathbf{1}_{\left\{\|y\|_{U}<1\right\}}\right) \nu(d y)\right\} .11\right)
\end{aligned}
$$

Using (5.7), (5.9), (5.10) we now estimate

$$
\begin{aligned}
& \int_{U \backslash\{0\}}\left(\tilde{\psi}_{N}^{\epsilon}(t, x+\gamma(x, a, y))-\tilde{\psi}_{N}^{\epsilon}(t, x)-\langle\gamma(x, a, y), D \psi(t, x)\rangle \mathbf{1}_{\left\{\|y\|_{U}<1\right\}}\right) \nu(d y) \\
& \leq \int_{\left\{0<\|y\|_{U}<r\right\}}(\psi(t, x+\gamma(x, a, y))-\psi(t, x)-\langle\gamma(x, a, y), D \psi(t, x)\rangle) \nu(d y) \\
& +\int_{\left\{\rho(y) \geq \frac{\epsilon}{c}\right\} \cup\left\{\|y\|_{U} \geq r\right\}} 4 \delta_{\epsilon} \nu(d y)+\int_{\left\{\rho(y) \geq \frac{\epsilon}{c}\right\} \cup\left\{\|y\|_{U} \geq r\right\}} 2 h_{\epsilon}\left(\left\|Q_{N}(x+\gamma(x, a, y))\right\|_{-1}\right) \nu(d y) \\
& \quad+\int_{\left\{\|y\|_{U} \geq r\right\}}\left(u(t, x+\gamma(x, a, y))-u(t, x)-\langle\gamma(x, a, y), D \psi(t, x)\rangle \mathbf{1}_{\left\{\|y\|_{U}<1\right\}}\right. \\
& \left.\quad+(\psi(t, x+\gamma(x, a, y))-u(t, x+\gamma(x, a, y))) \eta_{\epsilon}\left(\|\gamma(x, a, y)\|_{-1}\right)\right) \nu(d y) . \\
& \leq \int_{\left\{0<\|y\|_{U}<r\right\}}(\psi(t, x+\gamma(x, a, y))-\psi(t, x)-\langle\gamma(x, a, y), D \psi(t, x)\rangle) \nu(d y) \\
& +\int_{\left\{\|y\|_{U} \geq r\right\}}\left(u(t, x+\gamma(x, a, y))-u(t, x)-\langle\gamma(x, a, y), D \psi(t, x)\rangle \mathbf{1}_{\left.\left\{\|y\|_{U}<1\right\}\right) \nu(d y)+\sigma(\epsilon, N)}\right.
\end{aligned}
$$


where

$$
\lim _{\epsilon \rightarrow 0} \lim _{N \rightarrow+\infty} \sigma(\epsilon, N)=0 .
$$

To obtain the last inequality we used (4.10), Lebesgue dominated convergence theorem, and the fact that

$$
\lim _{\epsilon \rightarrow 0} \int_{\{\|y\| \geq r\}} \sup _{a \in \Gamma}\left\{|\psi(t, x+\gamma(x, a, y))-u(t, x+\gamma(x, a, y))| \eta_{\epsilon}\left(\|\gamma(x, a, y)\|_{-1}\right)\right\} \nu(d y)=0 .
$$

To see (5.13), by the dominated convergence theorem, it is enough to show that for every $y$, the integrand in (5.13) converges to 0 as $\epsilon \rightarrow 0$. Let $a_{\epsilon}$ be such that

$$
\begin{aligned}
& \left|\psi\left(t, x+\gamma\left(x, a_{\epsilon}, y\right)\right)-u\left(t, x+\gamma\left(x, a_{\epsilon}, y\right)\right)\right| \eta_{\epsilon}\left(\left\|\gamma\left(x, a_{\epsilon}, y\right)\right\|_{-1}\right) \\
& \quad \geq \sup _{a \in \Gamma}\left\{|\psi(t, x+\gamma(x, a, y))-u(t, x+\gamma(x, a, y))| \eta_{\epsilon}\left(\|\gamma(x, a, y)\|_{-1}\right)\right\}-\epsilon .
\end{aligned}
$$

If $\lim \inf _{\epsilon \rightarrow 0}\left\|\gamma\left(x, a_{\epsilon}, y\right)\right\|_{-1}>0$ we are done. If $\lim _{\epsilon_{n} \rightarrow 0}\left\|\gamma\left(x, a_{\epsilon_{n}}, y\right)\right\|_{-1}=0$ then, by precompactness of $\{\gamma(x, a, y): a \in \Gamma\}$ in $H$, there is a subsequence $\epsilon_{k_{k}}$ such that $\gamma\left(x, a_{\epsilon_{n_{k}}}, y\right) \rightarrow 0$ as $k \rightarrow \infty$. But then $\left|\psi\left(t, x+\gamma\left(x, a_{\epsilon_{n_{k}}}, y\right)\right)-u\left(t, x+\gamma\left(x, a_{\epsilon_{n_{k}}}, y\right)\right)\right| \rightarrow 0$ as $k \rightarrow \infty$ which proves the claim.

It now remains to plug (5.12) into (5.11) and let $N \rightarrow+\infty$ and then $\epsilon \rightarrow 0$ to obtain

$$
\psi_{t}(t, x)+\left\langle x, A^{*} D \varphi(t, x)\right\rangle+F_{r}\left(x, D \psi(t, x), D^{2} \psi(t, x), \psi(t, \cdot), u(t, \cdot)\right) \geq 0 .
$$

Remark 5.6. In many cases the integrability condition (4.2) can be strengthened. For instance, for a square integrable Lévy process we have $\rho(z)=\|z\|_{U}$ and

$$
\int_{H}\left((\rho(z))^{2} \nu(d z)<+\infty\right.
$$

In such cases we can consider important problems leading to unbounded viscosity solutions and the definition of viscosity solution must be modified to allow for unbounded test functions having proper growth and infinity. We leave these extensions to the readers. In particular, when (5.14) is satisfied, we can consider solutions which have quadratic growth at infinity and then use test functions which grow quadratically at infinity.

\section{Comparison Principle}

We begin with a finite dimensional parabolic maximum principle in a version suitable for integro-PDE. It can be deduced from basically the same arguments as the ones used in the proofs of Lemmas 7.3, 7.4, 7.7 of [41] or directly adapting the proof of the maximum principle for semicontinuous functions which can be found in [26]. 
Lemma 6.1. Let $u,-v$ be upper semicontinuous on $(0, T) \times \mathbb{R}^{N}$ and such that $u,-v$ are bounded from above. Let

$$
u(t, x)-v(s, y)-\frac{\|x-y\|^{2}}{2 \epsilon}-\frac{(t-s)^{2}}{2 \beta}
$$

have a strict global maximum at $(\bar{t}, \bar{s}, \bar{x}, \bar{y})$. Then there exist points $t_{k}, s_{k}, x_{k}, y_{k}$, functions $\varphi_{k}, \psi_{k}, \tilde{\varphi}_{k}, \tilde{\psi}_{k} \in U C^{2}\left([0, T] \times \mathbb{R}^{N}\right), \varphi_{k}, \tilde{\varphi}_{k}$ bounded from below, $\psi_{k}, \tilde{\psi}_{k}$ bounded from above, and $X, Y, X_{k}, Y_{k} \in \mathcal{S}(N)$ such that

$$
\begin{gathered}
\lim _{k \rightarrow+\infty}\left(t_{k}, s_{k}, x_{k}, y_{k}\right)=(\bar{t}, \bar{s}, \bar{x}, \bar{y}), \\
\lim _{k \rightarrow+\infty} u\left(t_{k}, x_{k}\right)=u(\bar{t}, \bar{x}), \quad \lim _{k \rightarrow+\infty} v\left(s_{k}, y_{k}\right)=v(\bar{s}, \bar{y}), \\
u-\varphi_{k} \quad \text { has a strict global maximum at }\left(t_{k}, x_{k}\right), \\
v-\psi_{k} \quad \text { has a strict global minimum at }\left(s_{k}, y_{k}\right), \\
\varphi_{k}\left(t_{k}, x_{k}\right)=\tilde{\varphi}_{k}\left(t_{k}, x_{k}\right), \varphi_{k} \leq \tilde{\varphi}_{k}, \\
\left|\varphi_{k}\right|+\left|\tilde{\varphi}_{k}\right| \leq C\left(1+\|\left. x\right|^{2}\right), \quad k \geq 1, \\
\left(\varphi_{k}\right)_{t}\left(t_{k}, x_{k}\right)=\left(\tilde{\varphi}_{k}\right)_{t}\left(t_{k}, x_{k}\right) \rightarrow \frac{\bar{t}-\bar{s}}{\beta} \quad \text { as } k \rightarrow+\infty, \\
D \varphi_{k}\left(t_{k}, x_{k}\right)=D \tilde{\varphi}_{k}\left(t_{k}, x_{k}\right) \rightarrow \frac{\bar{x}-\bar{y}}{\epsilon} \quad \text { as } k \rightarrow+\infty, \\
D^{2} \varphi_{k}\left(t_{k}, x_{k}\right) \leq X_{k} \rightarrow X \quad \text { as } k \rightarrow+\infty, \\
\psi_{k}\left(s_{k}, y_{k}\right)=\tilde{\psi}_{k}\left(s_{k}, y_{k}\right), \psi_{k} \geq \tilde{\psi}_{k}, \\
\left|\psi_{k}\right|+\left|\tilde{\psi}_{k}\right| \leq C\left(1+\|x\|^{2}\right), \quad k \geq 1, \\
D \psi_{k}\left(s_{k}, y_{k}\right)=D \tilde{\psi}_{k}\left(s_{k}, y_{k}\right) \rightarrow \frac{\bar{x}-\bar{y}}{\epsilon} \quad \text { as } k \rightarrow+\infty \\
D^{2} \psi_{k}\left(s_{k}, y_{k}\right) \geq Y_{k} \rightarrow Y \quad a s k \rightarrow+\infty, \\
\psi_{k}\left(s_{k}, y_{k}\right)=\left(\tilde{\psi}_{k}\right)_{t}\left(s_{k}, y_{k}\right) \rightarrow \frac{\bar{t}}{\beta} \quad \text { as } \rightarrow+\infty \\
\end{gathered}
$$

where

$$
-\frac{3}{\epsilon}\left(\begin{array}{ll}
I & 0 \\
0 & I
\end{array}\right) \leq\left(\begin{array}{cc}
X & 0 \\
0 & -Y
\end{array}\right) \leq \frac{3}{\epsilon}\left(\begin{array}{cc}
I & -I \\
-I & I
\end{array}\right) .
$$

and

$$
\begin{gathered}
\tilde{\varphi}_{k} \rightarrow \frac{\|\cdot-\bar{y}\|^{2}}{2 \epsilon}+\frac{(\cdot-\bar{s})^{2}}{2 \beta} \quad \text { as } k \rightarrow+\infty \text { in } U C_{\mathrm{loc}}^{2}\left([0, T] \times \mathbb{R}^{N}\right) \\
\tilde{\psi}_{k} \rightarrow-\frac{\|\bar{x}-\cdot\|^{2}}{2 \epsilon}-\frac{(\bar{t}-\cdot)^{2}}{2 \beta} \quad \text { as } k \rightarrow+\infty \text { in } U C_{\mathrm{loc}}^{2}\left([0, T] \times \mathbb{R}^{N}\right)
\end{gathered}
$$


Theorem 6.2. Let (4.2)-(4.9) be satisfied. Let $u \in B U C\left([0, T] \times H_{-1}\right)$ be a viscosity subsolution of (5.1) and $v \in B U C\left([0, T] \times H_{-1}\right)$ be a viscosity supersolution of (5.1). Suppose that $u(T, x) \leq v(T, x)$ for all $x \in H$. Then $u \leq v$.

Proof. We will show that the assumption $u \leq \leq v$ leads to a contradiction.

Step 1. Define $u^{\mu}(t, x)=u(t, x)-\frac{\mu}{t}$ and $v^{\mu}(s, y)=v(s, y)+\frac{\mu}{s}$ for $\mu>0$. For $\epsilon, \delta, \beta>0$ we consider the function

$$
\Phi(t, s, x, y)=u^{\mu}(t, x)-v^{\mu}(s, y)-\frac{\|x-y\|_{-1}^{2}}{2 \epsilon}-\delta\left(\|x\|^{2}+\|y\|^{2}\right)-\frac{(t-s)^{2}}{2 \beta} .
$$

Using perturbed optimization (see [31] and [29], page 424) we can find sequences $a_{n}, b_{n} \in$ $\mathbb{R}$, and $p_{n}, q_{n} \in H$ such that $\left|a_{n}\right|+\left|b_{n}\right|+\left\|p_{n}\right\|+\left\|q_{n}\right\| \leq \frac{1}{n}$ such that

$u^{\mu}(t, x)-v^{\mu}(s, y)-\frac{\|x-y\|_{-1}^{2}}{2 \epsilon}-\delta\left(\|x\|^{2}+\|y\|^{2}\right)-\frac{(t-s)^{2}}{2 \beta}+a_{n} t+b_{n} s+\left\langle B p_{n}, x\right\rangle+\left\langle B q_{n}, y\right\rangle$

achieves a strict global maximum at some point $(\bar{t}, \bar{s}, \bar{x}, \bar{y}) \in(0, T] \times(0, T] \times H \times H$. Standard considerations (see for instance [38]) yield

$$
\begin{array}{r}
\lim _{\beta \downarrow 0} \limsup _{n \rightarrow \infty} \frac{1}{2 \beta}(\bar{t}-\bar{s})^{2}=0 \quad \text { for every } \delta, \epsilon>0, \\
\lim _{\delta \downarrow 0} \limsup _{\beta \downarrow 0} \limsup _{n \rightarrow \infty} \delta\left(\|\bar{x}\|^{2}+\|\bar{y}\|^{2}\right)=0 \quad \text { for every } \epsilon>0, \\
\lim _{\epsilon \downarrow 0} \limsup _{\delta \downarrow 0} \limsup _{\beta \downarrow 0} \limsup _{n \rightarrow \infty} \frac{1}{2 \epsilon}\|\bar{x}-\bar{y}\|_{-1}^{2}=0 .
\end{array}
$$

If $u \not \leq v$ it thus follows from the above and the uniform continuity of $u, v$, that for sufficiently small $\mu, \epsilon, \delta, \beta>0$ and $n$ large enough, $0<\bar{t}, \bar{s}<T$.

Step 2. (Reduction to finite dimensions.) For $N \geq 1$ let us denote by $\tilde{H}_{N}$ the space $H_{N}$ equipped with the $\|\cdot\|_{-1}$ norm (which on $H_{N}$ is equivalent to $\|\cdot\|$ ). For a function $w \in C^{2}\left(H_{-1}\right)$ we will denote by $D_{H_{-1}} w, D_{H_{-1}}^{2} w$ its Frechet derivatives in this space. We now define

$$
\begin{aligned}
u_{1}(t, x)=u^{\mu}(t, x) & -\frac{\left\langle B Q_{N}(\bar{x}-\bar{y}), x\right\rangle}{\epsilon}-\frac{\left\|Q_{N}(x-\bar{x})\right\|_{-1}^{2}}{\epsilon} \\
& +\frac{\left\|Q_{N}(\bar{x}-\bar{y})\right\|_{-1}^{2}}{2 \epsilon}-\delta\|x\|^{2}+a_{n} t+\left\langle B p_{n}, x\right\rangle
\end{aligned}
$$

and

$$
v_{1}(s, y)=v^{\mu}(s, y)-\frac{\left\langle B Q_{N}(\bar{x}-\bar{y}), y\right\rangle}{\epsilon}+\frac{\left\|Q_{N}(y-\bar{y})\right\|_{-1}^{2}}{\epsilon}+\delta\|y\|^{2}-b_{n} s-\left\langle B q_{n}, y\right\rangle .
$$

Then

$$
u_{1}(t, x)-v_{1}(s, y)-\frac{1}{2 \epsilon}\left\|P_{N}(x-y)\right\|_{-1}^{2}-\frac{1}{2 \beta}(t-s)^{2}
$$


has a strict global maximum at $(\bar{t}, \bar{s}, \bar{x}, \bar{y})$. Setting

$$
\begin{aligned}
& \tilde{u}_{1}\left(t, x_{N}\right)=\sup _{x_{N}^{\perp} \in Q_{N} H} u_{1}\left(t, x_{N}+x_{N}^{\perp}\right), \\
& \tilde{v}_{1}\left(s, y_{N}\right)=\inf _{y_{N}^{\perp} \in Q_{N} H} v_{1}\left(s, y_{N}+y_{N}^{\perp}\right),
\end{aligned}
$$

we thus see that

$$
\tilde{u}_{1}\left(t, x_{N}\right)-\tilde{v}_{1}\left(s, y_{N}\right)-\frac{1}{2 \epsilon}\left\|x_{N}-y_{N}\right\|_{-1}^{2}-\frac{1}{2 \beta}(t-s)^{2}
$$

has a strict global maximum over $[0, T] \times[0, T] \times H_{N} \times H_{N}$ at $\left(\bar{t}, \bar{s}_{,} \bar{x}_{N}, \bar{y}_{N}\right)$. Moreover we also have $\tilde{u}_{1}\left(\bar{t}, \bar{x}_{N}\right)=u_{1}(\bar{t}, \bar{x}), \tilde{v}_{1}\left(\bar{s}, \bar{y}_{N}\right)=v_{1}(\bar{s}, \bar{y})$. Since $u, v \in B U C\left([0, T] \times H_{-1}\right)$ it also easily follows that $u, v \in U C_{\text {loc }}\left([0, T] \times \tilde{H}_{N}\right)$ (which here means that they are uniformly continuous on bounded subsets). We can now use Lemma 6.1 applied to $\tilde{u}_{1}, \tilde{v}_{1}$, in which $\mathbb{R}^{N}$ is replaced by $\tilde{H}_{N}$.

Step 3. (Non-local maximum principle.) Let then points $\left(t_{k}, s_{k}, x_{N, k}, y_{N, k}\right) \in(0, T) \times$ $(0, T) \times H_{N} \times H_{N}$, functions $\varphi_{k}, \psi_{k}, \tilde{\varphi}_{k}, \tilde{\psi}_{k} \in U C^{2}\left([0, T] \times \tilde{H}_{N}\right)$, and $X, Y, X_{k}, Y_{k} \in \mathcal{S}\left(\tilde{H}_{N}\right)$ be as in Lemma 6.1 for our case. Setting $\varphi_{k}(t, x)=\varphi_{k}\left(t, x_{N}\right), \psi_{k}(s, y)=\psi_{k}\left(s, y_{N}\right), \tilde{\varphi}_{k}(t, x)=$ $\tilde{\varphi}_{k}\left(t, x_{N}\right), \tilde{\psi}_{k}(s, y)=\tilde{\psi}_{k}\left(s, y_{N}\right)$ we can consider them as functions in $U C^{2}\left([0, T] \times H_{-1}\right)$ and $X, Y, X_{k}, Y_{k}$ as elements of $\mathcal{S}\left(H_{-1}\right)$, such that $X=P_{N} X P_{N}, Y=P_{N} Y P_{N}, X_{k}=$ $P_{N} X_{k} P_{N}, Y_{k}=P_{N} Y_{k} P_{N}$ as operators in $\mathcal{L}\left(H_{-1}\right)$. (We remark that in (6.2)-(6.5) we now have $D_{\tilde{H}_{N}}, D_{\tilde{H}_{N}}^{2}$.) Since the norm in $\tilde{H}_{N}$ is equivalent to the norm in $H$, the convergence (6.1) holds in $\mathbb{R} \times \mathbb{R} \times H \times H$, the convergences in (6.2), (6.4) hold in $H$, and the convergences in $(6.3),(6.5)$ hold in $\mathcal{L}(H)$. Using the fact that $D \varphi_{k}=B D_{H_{-1}} \varphi_{k}, D^{2} \varphi_{k}=$ $B D_{H_{-1}}^{2} \varphi_{k}, D \psi_{k}=B D_{H_{-1}} \psi_{k}, D^{2} \psi_{k}=B D_{H_{-1}}^{2} \psi_{k}$, and the same is also true for functions $\tilde{\varphi}_{k}, \tilde{\psi}_{k}$, we thus have that $\varphi_{k}, \psi_{k}, \tilde{\varphi}_{k}, \tilde{\psi}_{k}$ are good test functions and moreover we have

$$
\begin{gathered}
\lim _{k \rightarrow+\infty}\left(t_{k}, s_{k}, x_{N, k}, y_{N, k}\right)=\left(\bar{t}, \bar{s}, \bar{x}_{N}, \bar{y}_{N}\right) \quad \text { in } \mathbb{R} \times \mathbb{R} \times H \times H, \\
\lim _{k \rightarrow+\infty} u_{1}\left(t_{k}, x_{N, k}\right)=u_{1}(\bar{t}, \bar{x}), \quad \lim _{k \rightarrow+\infty} \tilde{v}_{1}\left(s_{k}, y_{N, k}\right)=v_{1}(\bar{s}, \bar{y}),
\end{gathered}
$$

$\tilde{u}_{1}-\varphi_{k}$ has a strict global maximum over $(0, T) \times H_{N}$ at $\left(t_{k}, x_{N, k}\right)$,

$\tilde{v}_{1}-\psi_{k}$ has a strict global minimum over $(0, T) \times H_{N}$ at $\left(s_{k}, y_{N, k}\right)$,

$$
\begin{gathered}
\varphi_{k}\left(t_{k}, x_{N, k}\right)=\tilde{\varphi}_{k}\left(t_{k}, x_{N, k}\right), \varphi_{k} \leq \tilde{\varphi}_{k}, \\
\left|\varphi_{k}\right|+\left|\tilde{\varphi}_{k}\right| \leq C\left(1+\|x\|^{2}\right), \quad k \geq 1 \\
\left(\varphi_{k}\right)_{t}\left(t_{k}, x_{N, k}\right)=\left(\tilde{\varphi}_{k}\right)_{t}\left(t_{k}, x_{N, k}\right) \rightarrow \frac{\bar{t}-\bar{s}}{\beta} \quad \text { as } k \rightarrow+\infty, \\
B^{-1} D \varphi_{k}\left(t_{k}, x_{N, k}\right)=B^{-1} D \tilde{\varphi}_{k}\left(t_{k}, x_{N, k}\right) \rightarrow \frac{\bar{x}_{N}-\bar{y}_{N}}{\epsilon} \quad \text { in } H \text { as } k \rightarrow+\infty,
\end{gathered}
$$




$$
\begin{gathered}
D^{2} \varphi_{k}\left(t_{k}, x_{N, k}\right) \leq X_{k} \rightarrow X \quad \text { in } \mathcal{L}(H) \text { as } k \rightarrow+\infty, \\
\psi_{k}\left(s_{k}, y_{N, k}\right)=\tilde{\psi}_{k}\left(s_{k}, y_{N, k}\right), \psi_{k} \geq \tilde{\psi}_{k}, \\
\left|\psi_{k}\right|+\left|\tilde{\psi}_{k}\right| \leq C\left(1+\|x\|^{2}\right), \quad k \geq 1 \\
\left(\psi_{k}\right)_{t}\left(s_{k}, y_{N, k}\right)=\left(\tilde{\psi}_{k}\right)_{t}\left(s_{k}, y_{N, k}\right) \rightarrow \frac{\bar{t}-\bar{s}}{\beta} \quad \text { as } k \rightarrow+\infty, \\
B^{-1} D \psi_{k}\left(s_{k}, y_{N, k}\right)=B^{-1} D \tilde{\psi}_{k}\left(s_{k}, y_{N, k}\right) \rightarrow \frac{\bar{x}_{N}-\bar{y}_{N}}{\epsilon} \quad \text { in } H \text { as } k \rightarrow+\infty, \\
D^{2} \psi_{k}\left(s_{k}, y_{N, k}\right) \geq Y_{k} \rightarrow Y \quad \text { in } \mathcal{L}(H) \text { as } k \rightarrow+\infty,
\end{gathered}
$$

where

$$
-\frac{3}{\epsilon}\left(\begin{array}{cc}
B & 0 \\
0 & B
\end{array}\right) \leq\left(\begin{array}{cc}
X & 0 \\
0 & -Y
\end{array}\right) \leq \frac{3}{\epsilon}\left(\begin{array}{cc}
B & -B \\
-B & B
\end{array}\right) .
$$

as operators in $\mathcal{L}(H \times H)$, and

$$
\begin{gathered}
\tilde{\varphi}_{k} \rightarrow \frac{\left\|(\cdot-\bar{y})_{N}\right\|_{-1}^{2}}{2 \epsilon}+\frac{(\cdot-\bar{s})^{2}}{2 \beta} \quad \text { as } k \rightarrow+\infty \text { in } U C_{\mathrm{loc}}^{2}([0, T] \times H), \\
\tilde{\psi}_{k} \rightarrow-\frac{\left\|(\bar{x}-\cdot)_{N}\right\|_{-1}^{2}}{2 \epsilon}-\frac{(\bar{t}-\cdot)^{2}}{2 \beta} \text { as } k \rightarrow+\infty \text { in } U C_{\mathrm{loc}}^{2}([0, T] \times H) .
\end{gathered}
$$

(Convergence in $U C_{\text {loc }}^{2}([0, T] \times H)$ means convergence in $U C^{2}([0, T] \times H)$ on bounded subsets of $[0, T] \times H$.)

Step 4. (Back to infinite dimensions.) We now use again perturbed optimization to obtain that for every $j \geq 1$ there exist $a_{k}^{j}, b_{k}^{j} \in \mathbb{R}$, and $p_{k}^{j}, q_{k}^{j} \in H$ such that $\left|a_{k}^{j}\right|+\left|b_{k}^{j}\right|+$ $\left\|p_{k}^{j}\right\|+\left\|q_{k}^{j}\right\| \leq \frac{1}{j}$ and points $\left(t_{k}^{j}, s_{k}^{j}, x_{k}^{j}, y_{k}^{j}\right) \in(0, T) \times(0, T) \times H \times H$ such that

$$
\begin{aligned}
& u_{1}(t, x)-\varphi_{k}(t, x)-a_{k}^{j} t-\left\langle B p_{k}^{j}, x\right\rangle \text { has a global maximum over }(0, T) \times H \text { at }\left(t_{k}^{j}, x_{k}^{j}\right), \\
& v_{1}(s, y)-\psi_{k}(s, y)-b_{k}^{j} s-\left\langle B q_{k}^{j}, y\right\rangle \text { has a global minimum over }(0, T) \times H \text { at }\left(s_{k}^{j}, y_{k}^{j}\right) .
\end{aligned}
$$

Rather standard arguments (see for instance [27] or [46], page 261) allow us now to conclude that $\left(t_{k}^{j}, s_{k}^{j},\left(x_{k}^{j}\right)_{N},\left(y_{k}^{j}\right)_{N}\right) \rightarrow\left(t_{k}, s_{k}, x_{N, k}, y_{N, k}\right)$ as $j \rightarrow+\infty$ and show that there exists a subsequence $j_{k}$ such that $\left(t_{k}^{j_{k}}, s_{k}^{j_{k}}, x_{k}^{j_{k}}, y_{k}^{j_{k}}\right) \rightarrow(\bar{t}, \bar{s}, \bar{x}, \bar{y})$ as $k \rightarrow+\infty$ which we then easily can choose to satisfy

$$
\begin{gathered}
\left(\varphi_{k}\right)_{t}\left(t_{k}^{j_{k}}, x_{k}^{j_{k}}\right) \rightarrow \frac{\bar{t}-\bar{s}}{\beta} \quad \text { as } k \rightarrow+\infty, \\
B^{-1} D \varphi_{k}\left(t_{k}^{j_{k}}, x_{k}^{j_{k}}\right) \rightarrow \frac{\bar{x}_{N}-\bar{y}_{N}}{\epsilon} \quad \text { in } H \text { as } k \rightarrow+\infty, \\
\left(\psi_{k}\right)_{t}\left(s_{k}^{j_{k}}, y_{k}^{j_{k}}\right) \rightarrow \frac{\bar{t}-\bar{s}}{\beta} \quad \text { as } k \rightarrow+\infty,
\end{gathered}
$$




$$
\begin{gathered}
B^{-1} D \psi_{k}\left(s_{k}^{j_{k}}, y_{k}^{j_{k}}\right) \rightarrow \frac{\bar{x}_{N}-\bar{y}_{N}}{\epsilon} \quad \text { in } H \text { as } k \rightarrow+\infty, \\
D^{2} \varphi_{k}\left(t_{k}^{j_{k}}, x_{k}^{j_{k}}\right)-X_{k} \leq \omega(k) I, \\
D^{2} \psi_{k}\left(s_{k}^{j_{k}}, y_{k}^{j_{k}}\right)-Y_{k} \geq-\omega(k) I,
\end{gathered}
$$

where $\lim _{k \rightarrow+\infty} \omega(k)=0$, and, denoting $\hat{x}_{N, k}=x_{N, k}+\left(x_{k}^{j_{k}}\right)_{N}^{\perp}, \hat{y}_{N, k}=y_{N, k}+\left(y_{k}^{j_{k}}\right)_{N}^{\perp}$,

$$
\begin{gathered}
\sup _{a \in \Gamma} \int_{\left\{0<\|y\|_{U}<r\right\}} \mid\left(\varphi_{k}\left(t_{k}^{j_{k}}, x_{k}^{j_{k}}+\gamma\left(x_{k}^{j_{k}}, a, y\right)\right)-\varphi_{k}\left(t_{k}^{j_{k}}, x_{k}^{j_{k}}\right)-\left\langle\gamma\left(x_{k}^{j_{k}}, a, y\right), D \varphi_{k}\left(t_{k}^{j_{k}}, x_{k}^{j_{k}}\right)\right\rangle\right) \\
-\left(\varphi_{k}\left(t_{k}, \hat{x}_{N, k}+\gamma\left(\hat{x}_{N, k}, a, y\right)\right)-\varphi_{k}\left(t_{k}, \hat{x}_{N, k}\right)-\left\langle\gamma\left(\hat{x}_{N, k}, a, y\right), D \varphi_{k}\left(t_{k}, \hat{x}_{N, k}\right)\right\rangle\right) \mid \nu(d y) \\
\rightarrow 0 \quad \text { as } k \rightarrow+\infty, \\
\sup _{a \in \Gamma} \int_{\left\{0<\|y\|_{U}<r\right\}} \mid\left(\psi_{k}\left(s_{k}^{j_{k}}, y_{k}^{j_{k}}+\gamma\left(y_{k}^{j_{k}}, a, y\right)\right)-\psi_{k}\left(s_{k}^{j_{k}}, y_{k}^{j_{k}}\right)-\left\langle\gamma\left(y_{k}^{j_{k}}, a, y\right), D \psi_{k}\left(s_{k}^{j_{k}}, y_{k}^{j_{k}}\right)\right\rangle\right) \\
-\left(\psi_{k}\left(s_{k}, \hat{y}_{N, k}+\gamma\left(\hat{y}_{N, k}, a, y\right)\right)-\psi_{k}\left(s_{k}, \hat{y}_{N, k}\right)-\left\langle\gamma\left(\hat{y}_{N, k}, a, y\right), D \psi_{k}\left(s_{k}, \hat{y}_{N, k}\right)\right\rangle\right) \mid \nu(d y) \\
\rightarrow 0 \quad \text { as } k \rightarrow+\infty .
\end{gathered}
$$

(Convergences (6.35) and (6.36) follow from uniform continuity of $\varphi_{k}, \psi_{k}$, and its derivatives, and (4.4), (4.7).)

Thus, using (4.3), (6.14), (6.15), (6.17), (6.32), (6.25) and (6.35), (recall that $\varphi_{k}\left(t_{k}, \hat{x}_{N, k}\right)=$ $\left.\tilde{\varphi}_{k}\left(t_{k}, x_{N, k}\right)\right)$ we obtain that for every $a \in \Gamma$ and $0<r<1$

$$
\begin{aligned}
& \int_{\left\{0<\|y\|_{U}<r\right\}}\left(\varphi_{k}\left(t_{k}^{j_{k}}, x_{k}^{j_{k}}+\gamma\left(x_{k}^{j_{k}}, a, y\right)\right)-\varphi_{k}\left(t_{k}^{j_{k}}, x_{k}^{j_{k}}\right)-\left\langle\gamma\left(x_{k}^{j_{k}}, a, y\right), D \varphi_{k}\left(t_{k}^{j_{k}}, x_{k}^{j_{k}}\right)\right\rangle\right) \nu(d y) \\
& \leq \int_{\left\{0<\|y\|_{U}<r\right\}} \begin{array}{r}
\left(\varphi_{k}\left(t_{k}, \hat{x}_{N, k}+\gamma\left(\hat{x}_{N, k}, a, y\right)\right)-\varphi_{k}\left(t_{k}, \hat{x}_{N, k}\right)-\left\langle\gamma\left(\hat{x}_{N, k}, a, y\right), D \varphi_{k}\left(t_{k}, \hat{x}_{N, k}\right)\right\rangle\right) \nu(d y) \\
\quad+\omega_{1}(k)
\end{array} \\
& \leq \int_{\left\{0<\|y\|_{U}<r\right\}} \begin{array}{c}
\left(\tilde{\varphi}_{k}\left(t_{k}, \hat{x}_{N, k}+\gamma\left(\hat{x}_{N, k}, a, y\right)\right)-\tilde{\varphi}_{k}\left(t_{k}, \hat{x}_{N, k}\right)-\left\langle\gamma\left(\hat{x}_{N, k}, a, y\right), D \tilde{\varphi}_{k}\left(t_{k}, \hat{x}_{N, k}\right)\right\rangle\right) \nu(d y) \\
\quad+\omega_{1}(k) \\
\leq \int_{\left\{0<\|y\|_{U}<r\right\}}\left(\frac{\left\|(\bar{x}+\gamma(\bar{x}, a, y))_{N}-\bar{y}_{N}\right\|_{-1}^{2}}{2 \epsilon}-\frac{\left\|\bar{x}_{N}-\bar{y}_{N}\right\|_{-1}^{2}}{2 \epsilon}-\left\langle\gamma(\bar{x}, a, y), \frac{B\left(\bar{x}_{N}-\bar{y}_{N}\right)}{\epsilon}\right\rangle\right) \nu(d y) \\
+\omega_{2}(k) \\
\left.=\int_{\left\{0<\|y\|_{U}<r\right\}} \frac{\left\|(\gamma(\bar{x}, a, y))_{N}\right\|_{-1}^{2} \nu(d y)+\omega_{2}(k) \leq \omega_{2}(k)+\omega_{3}(r),}{2 \epsilon} \nu .37\right)
\end{array}
\end{aligned}
$$

where $\lim _{k \rightarrow+\infty} \omega_{2}(k)=0, \lim _{r \rightarrow 0} \omega_{3}(r)=0$ for fixed $\epsilon, \delta, \beta, n, N$, and $\omega_{2}(k), \omega_{3}(r)$ are independent of $a \in \Gamma$ and $\omega_{2}(k)$ also of $0<r<1$. 
Similarly, for every $a \in \Gamma$ and $0<r<1$,

$$
\begin{gathered}
\int_{\left\{0<\|y\|_{U}<r\right\}}\left(\psi_{k}\left(s_{k}^{j_{k}}, y_{k}^{j_{k}}+\gamma\left(y_{k}^{j_{k}}, a, y\right)\right)-\psi_{k}\left(s_{k}^{j_{k}}, y_{k}^{j_{k}}\right)-\left\langle\gamma\left(y_{k}^{j_{k}}, a, y\right), D \psi_{k}\left(s_{k}^{j_{k}}, y_{k}^{j_{k}}\right)\right\rangle\right) \nu(d y) \\
\geq-\omega_{2}(k)-\omega_{3}(r) .
\end{gathered}
$$

Step 5. (Viscosity inequalities.) Using Definition 5.4 of viscosity subsolution (recall (6.27) and the definition of $u_{1}$ ), and (6.37), we thus obtain

$$
\begin{aligned}
& -\frac{\mu}{\left(t_{k}^{j_{k}}\right)^{2}}+\left(\varphi_{k}\right)_{t}\left(t_{k}^{j_{k}}, x_{k}^{j_{k}}\right)-a_{n}+a_{k}^{j_{k}} \\
& -\left\langle x_{k}^{j_{k}}, A^{*}\left(D \varphi_{k}\left(t_{k}^{j_{k}}, x_{k}^{j_{k}}\right)+\frac{B Q_{N}(\bar{x}-\bar{y})}{\epsilon}+\frac{2 B Q_{N}\left(x_{k}^{j_{k}}-\bar{x}\right)}{\epsilon}-B p_{n}+B p_{k}^{j_{k}}\right)\right\rangle \\
& +\inf _{a \in \Gamma}\left\{\frac{1}{2} \operatorname{Tr}\left(\sigma\left(x_{k}^{j_{k}}, a\right) \sigma^{*}\left(x_{k}^{j_{k}}, a\right)\left(D^{2} \varphi_{k}\left(t_{k}^{j_{k}}, x_{k}^{j_{k}}\right)+2 \delta I+\frac{2 B Q_{N}}{\epsilon}\right)\right)+f\left(x_{k}^{j_{k}}, a\right)\right. \\
& +\left\langle b\left(x_{k}^{j_{k}}, a\right), D \varphi_{k}\left(t_{k}^{j_{k}}, x_{k}^{j_{k}}\right)+\frac{B Q_{N}(\bar{x}-\bar{y})}{\epsilon}+2 \delta x_{k}^{j_{k}}+\frac{2 B Q_{N}\left(x_{k}^{j_{k}}-\bar{x}\right)}{\epsilon}-B p_{n}+B p_{k}^{j_{k}}\right\rangle \\
& +\int_{\left\{0<\|y\|_{U}<r\right\}}\left(\frac{\left\|Q_{N} \gamma\left(x_{k}^{j_{k}}, a, y\right)\right\|_{-1}^{2}}{\epsilon}+\delta\left\|\gamma\left(x_{k}^{j_{k}}, a, y\right)\right\|^{2}\right) \nu(d y)+\omega_{2}(k)+\omega_{3}(r) \\
& +\int_{\left\{\|y\|_{U} \geq r\right\}}\left(u\left(t_{k}^{j_{k}}, x_{k}^{j_{k}}+\gamma\left(x_{k}^{j_{k}}, a, y\right)\right)-u\left(t_{k}^{j_{k}}, x_{k}^{j_{k}}\right)-\left\langle\gamma\left(x_{k}^{j_{k}}, a, y\right),\right.\right. \\
& \left.\left.\left.\quad D \varphi_{k}\left(t_{k}^{j_{k}}, x_{k}^{j_{k}}\right)+\frac{B Q_{N}(\bar{x}-\bar{y})}{\epsilon}+2 \delta x_{k}^{j_{k}}+\frac{2 B Q_{N}\left(x_{k}^{j_{k}}-\bar{x}\right)}{\epsilon}-B p_{n}+B p_{k}^{j_{k}}\right\rangle \mathbf{1}_{\left\{\|y\|_{U}<1\right\}}\right) \nu(d y)\right\} \geq 0 .
\end{aligned}
$$

Since

$$
\sup _{a \in \Gamma, k \geq 1} \int_{\left\{0<\|y\|_{U}<r\right\}}\left(\frac{\left\|Q_{N} \gamma\left(x_{k}^{j_{k}}, a, y\right)\right\|_{-1}^{2}}{\epsilon}+\delta\left\|\gamma\left(x_{k}^{j_{k}}, a, y\right)\right\|^{2}\right) \nu(d y) \leq \omega_{4}(r),
$$

where $\lim _{r \rightarrow 0} \omega_{4}(r)=0$ for fixed $\epsilon, \delta, \beta, n, N$, letting $k \rightarrow+\infty$ in (6.39) and using (6.18), (6.29)-(6.34) we obtain

$$
\begin{aligned}
& -\frac{\bar{t}-\bar{s}}{T^{2}}+\frac{a_{n}}{\beta} \\
- & \left\langle\bar{x}, A^{*}\left(\frac{B(\bar{x}-\bar{y})}{\epsilon}-B p_{n}\right)\right\rangle \\
+ & \inf _{a \in \Gamma}\left\{\frac{1}{2} \operatorname{Tr}\left(\sigma(\bar{x}, a) \sigma^{*}(\bar{x}, a)\left(X+2 \delta I+\frac{2 B Q_{N}}{\epsilon}\right)\right)+f(\bar{x}, a)\right. \\
+ & \left\langle b(\bar{x}, a), \frac{B(\bar{x}-\bar{y})}{\epsilon}+2 \delta \bar{x}-B p_{n}\right\rangle+\omega_{5}(\epsilon, \delta, \beta, n, N ; r) \\
+ & \int_{\{\|y\| \geq r\}}(u(\bar{t}, \bar{x}+\gamma(\bar{x}, a, y))-u(\bar{t}, \bar{x})-\langle\gamma(\bar{x}, a, y), \\
& \left.\left.\left.\frac{B(\bar{x}-\bar{y})}{\epsilon}+2 \delta \bar{x}-B p_{n}\right\rangle \mathbf{1}_{\left\{\|y\|_{U}<1\right\}}\right) \nu(d y)\right\} \geq 0,
\end{aligned}
$$


where $\lim _{r \rightarrow 0} \omega_{5}(\epsilon, \delta, \beta, n, N ; r)=0$.

Similar computation, using Definition 5.4 of viscosity supersolution (recall (6.28) and the definition of $v_{1}$ ) and (6.38), gives us

$$
\begin{aligned}
& \frac{\mu}{T^{2}}+\frac{\bar{t}-\bar{s}}{\beta}+b_{n} \\
& -\left\langle\bar{y}, A^{*}\left(\frac{B(\bar{x}-\bar{y})}{\epsilon}+B q_{n}\right)\right\rangle \\
& +\inf _{a \in \Gamma}\left\{\frac{1}{2} \operatorname{Tr}\left(\sigma(\bar{y}, a) \sigma^{*}(\bar{y}, a)\left(Y-2 \delta I-\frac{2 B Q_{N}}{\epsilon}\right)\right)+f(\bar{y}, a)\right. \\
& +\left\langle b(\bar{y}, a), \frac{B(\bar{x}-\bar{y})}{\epsilon}-2 \delta \bar{y}+B q_{n}\right\rangle-\omega_{5}(\epsilon, \delta, \beta, n, N ; r) \\
& +\int_{\left\{\|y\|_{U} \geq r\right\}}(v(\bar{s}, \bar{y}+\gamma(\bar{y}, a, y))-v(\bar{s}, \bar{y})-\langle\gamma(\bar{y}, a, y), \\
& \left.\left.\left.\quad \frac{B(\bar{x}-\bar{y})}{\epsilon}-2 \delta \bar{y}+B q_{n}\right\rangle \mathbf{1}_{\left\{\|y\|_{U}<1\right\}}\right) \nu(d y)\right\} \leq 0 .
\end{aligned}
$$

Step 6. (Estimates.) Now, if $r \leq\|y\|_{U}$,

$$
\begin{aligned}
& u(\bar{t}, \bar{x}+\gamma(\bar{x}, a, y))-v(\bar{s}, \bar{y}+\gamma(\bar{y}, a, y)) \\
& -\frac{\|\bar{x}+\gamma(\bar{x}, a, y)-(\bar{y}+\gamma(\bar{y}, a, y))\|_{-1}^{2}}{2 \epsilon}-\delta\left(\|\bar{x}+\gamma(\bar{x}, a, y)\|^{2}+\|\bar{y}+\gamma(\bar{y}, a, y)\|^{2}\right) \\
& +\left\langle B p_{n}, \bar{x}+\gamma(\bar{x}, a, y)\right\rangle+\left\langle B q_{n}, \bar{y}+\gamma(\bar{y}, a, y)\right\rangle \\
& \leq u(\bar{t}, \bar{x})-v(\bar{s}, \bar{y})-\frac{\|\bar{x}-\bar{y}\|_{-1}^{2}}{2 \epsilon}-\delta\left(\|\bar{x}\|^{2}+\|\bar{y}\|^{2}\right)+\left\langle B p_{n}, \bar{x}\right\rangle+\left\langle B q_{n}, \bar{y}\right\rangle,
\end{aligned}
$$

which implies, by (4.4) and (4.7), that for every $a \in \Gamma, y \in U$

$$
\begin{aligned}
& u(\bar{t}, \bar{x}+\gamma(\bar{x}, a, y))-u(\bar{t}, \bar{x})-\left\langle\gamma(\bar{x}, a, y), \frac{B(\bar{x}-\bar{y})}{\epsilon}+2 \delta \bar{x}-B p_{n}\right\rangle \\
& -\left(v(\bar{s}, \bar{y}+\gamma(\bar{y}, a, y))-v(\bar{s}, \bar{y})-\left\langle\gamma(\bar{y}, a, y), \frac{B(\bar{x}-\bar{y})}{\epsilon}-2 \delta \bar{y}+B q_{n}\right\rangle\right) \\
& \leq \frac{\|\gamma(\bar{x}, a, y)-\gamma(\bar{y}, a, y)\|_{-1}^{2}}{2 \epsilon}+\delta\left(\|\gamma(\bar{x}, a, y)\|^{2}+\|\gamma(\bar{y}, a, y)\|^{2}\right) \\
& \leq C\left(\frac{\|\bar{x}-\bar{y}\|_{-1}^{2}}{2 \epsilon}+\delta\left(1+\|\bar{x}\|^{2}+\|\bar{y}\|^{2}\right)\right)(\rho(y))^{2} .
\end{aligned}
$$

If $\|y\|_{U} \geq 1,(6.41)$ also implies that

$$
\begin{aligned}
& u(\bar{t}, \bar{x}+\gamma(\bar{x}, a, y))-u(\bar{t}, \bar{x})-(v(\bar{s}, \bar{y}+\gamma(\bar{y}, a, y))-v(\bar{s}, \bar{y})) \\
& \leq\left(\frac{\|\bar{x}-\bar{y}\|_{-1}^{2}}{2 \epsilon}+\delta\left(1+\|\bar{x}\|^{2}+\|\bar{y}\|^{2}\right)+\frac{1}{n}(1+\|\bar{x}\|+\|\bar{y}\|)\right) C(y)
\end{aligned}
$$


for some $C(y) \geq 0$. It is easy to see using (6.6)-(6.8) that we can choose a sequence $\left(\epsilon_{k}, \delta_{k}, \beta_{k}, n_{k}\right)$ such that, denoting $\left(\bar{t}_{k}, \bar{s}_{k}, \bar{x}_{k}, \bar{y}_{k}\right)$ to be the point $(\bar{t}, \bar{s}, \bar{x}, \bar{y})$ corresponding to $\left(\epsilon_{k}, \delta_{k}, \beta_{k}, n_{k}\right)$, we have

$$
\begin{gathered}
\limsup \limsup _{\delta \downarrow 0} \limsup _{\beta \downarrow 0} \limsup _{n \rightarrow \infty} \int_{\left\{\|y\|_{U} \geq 1\right\}} \sup _{a \in \Gamma}(u(\bar{t}, \bar{x}+\gamma(\bar{x}, a, y))-u(\bar{t}, \bar{x}) \\
-(v(\bar{s}, \bar{y}+\gamma(\bar{y}, a, y))-v(\bar{s}, \bar{y}))) \nu(d y) \\
=\lim _{k \rightarrow \infty} \int_{\left\{\|y\|_{U} \geq 1\right\}} \sup _{a \in \Gamma}\left(u\left(\bar{t}_{k}, \bar{x}_{k}+\gamma\left(\bar{x}_{k}, a, y\right)\right)-u\left(\bar{t}_{k}, \bar{x}_{k}\right)\right. \\
\left.-\left(v\left(\bar{s}_{k}, \bar{y}_{k}+\gamma\left(\bar{y}_{k}, a, y\right)\right)-v\left(\bar{s}_{k}, \bar{y}_{k}\right)\right)\right) \nu(d y)
\end{gathered}
$$

and such that

$$
\lim _{k \rightarrow \infty}\left(\frac{1}{n_{k}}\left(\left\|\bar{x}_{k}\right\|+\left\|\bar{y}_{k}\right\|\right)+\delta_{k}\left(\left\|\bar{x}_{k}\right\|^{2}+\left\|\bar{y}_{k}\right\|^{2}\right)+\frac{\left\|\bar{x}_{k}-\bar{y}_{k}\right\|_{-1}^{2}}{2 \epsilon_{k}}\right)=0 .
$$

Then, (6.42), (6.43), (6.44), and the Lebesgue dominated convergence theorem, imply

$$
\begin{array}{r}
\limsup \limsup _{\delta \downarrow 0} \limsup _{\beta \downarrow 0} \limsup _{n \rightarrow \infty} \int_{\left\{\|y\|_{U} \geq 1\right\}} \sup _{a \in \Gamma}(u(\bar{t}, \bar{x}+\gamma(\bar{x}, a, y))-u(\bar{t}, \bar{x}) \\
-(v(\bar{s}, \bar{y}+\gamma(\bar{y}, a, y))-v(\bar{s}, \bar{y}))) \nu(d y) \leq 0 .
\end{array}
$$

Thus, subtracting (6.40) from (6.39), using (2.1), (4.2)-(4.7), (4.9), the fact that $X, Y$ satisfy (6.24), and standard properties of traces, we obtain

$$
\begin{gathered}
\frac{2 \mu}{T^{2}} \leq C\left(\frac{\|\bar{x}-\bar{y}\|_{-1}^{2}}{2 \epsilon}+\delta\left(1+\|\bar{x}\|^{2}+\|\bar{y}\|^{2}\right)\right)+\omega_{6}(\epsilon, \delta, \beta ; n, N, r) \\
+\inf _{a \in \Gamma}\left\{\int_{\left\{\|y\|_{U} \geq r\right\}}(u(\bar{t}, \bar{x}+\gamma(\bar{x}, a, y))-u(\bar{t}, \bar{x})-\langle\gamma(\bar{x}, a, y),\right. \\
\left.\left.\left.\frac{B(\bar{x}-\bar{y})}{\epsilon}+2 \delta \bar{x}-B p_{n}\right\rangle \mathbf{1}_{\left\{\|y\|_{U}<1\right\}}\right) \nu(d y)\right\} \\
-\inf _{a \in \Gamma}\left\{\int_{\left\{\|y\|_{U} \geq r\right\}}(v(\bar{s}, \bar{y}+\gamma(\bar{y}, a, y))-v(\bar{s}, \bar{y})-\langle\gamma(\bar{y}, a, y),\right. \\
\left.\left.\left.\frac{B(\bar{x}-\bar{y})}{\epsilon}-2 \delta \bar{y}+B q_{n}\right\rangle \mathbf{1}_{\left\{\|y\|_{U}<1\right\}}\right) \nu(d y)\right\},
\end{gathered}
$$

where $\lim \sup _{n \rightarrow \infty} \lim \sup _{N \rightarrow \infty} \lim \sup _{r \downarrow 0} \omega_{6}(\epsilon, \delta, \beta ; n, N, r)=0$, which, upon using (6.6)(6.8) (6.41) and (6.45), yields

$$
\begin{aligned}
& \frac{2 \mu}{T^{2}} \leq \omega_{7}(\epsilon, \delta, \beta, n, N, r) \\
& +\int_{\left\{\|y\|_{U} \geq 1\right\}} \sup _{a \in \Gamma}(u(\bar{t}, \bar{x}+\gamma(\bar{x}, a, y))-u(\bar{t}, \bar{x})-(v(\bar{s}, \bar{y}+\gamma(\bar{y}, a, y))-v(\bar{s}, \bar{y})) \nu(d y) \\
& \leq \omega_{8}(\epsilon, \delta, \beta, n, N, r),
\end{aligned}
$$


where

$$
\limsup _{\epsilon \downarrow 0} \limsup _{\delta \downarrow 0} \limsup _{\beta \downarrow 0} \limsup _{n \rightarrow \infty} \limsup _{N \rightarrow \infty} \limsup _{r \downarrow 0} \omega_{8}(\epsilon, \delta, \beta, n, N, r)=0 .
$$

This contradiction shows that we must have $u \leq v$.

Remark 6.3. If (4.3) $i$ replaced by (5.14) in Theorem 6.2 then its proof can be made substantially simpler. In this case there is no need to use Definition 5.4 (and thus Lemma 5.5 is not necessary), and in the proof after (6.40) there is no need to consider separately

the cases $\|y\|_{U}<1$ and $\|y\|_{U} \geq 1$. It is enough to use (6.41) in both cases directly after (6.40) was subtracted from (6.39). A splitting argument to estimate the integral terms for $r \leq\|y\|_{U}<1$ and $\|y\|_{U} \geq 1$ separately in the proof of comparison theorem was used in [41].

\section{References}

[1] H. Abels, and M. Kassmann, An analytic approach to purely nonlocal Bellman equations arising in models of stochastic control, J. Differential Equations 236 (2007), no. $1,29-56$.

[2] N. Alibaud, Existence, uniqueness and regularity for nonlinear parabolic equations with nonlocal terms, NoDEA Nonlinear Differential Equations Appl. 14 (2007), no. 3-4, 259-289.

[3] N. Alibaud, and C. Imbert, Fractional semi-linear parabolic equations with unbounded data, Trans. Amer. Math. Soc. 361 (2009), no. 5, 2527-2566.

[4] O. Alvarez, and A. Tourin, Viscosity solutions of nonlinear integro-differential equations, Ann. Inst. H. Poincaré Anal. Non Linéaire 13 (1996), no. 3, 293-317.

[5] A. L. Amadori, Nonlinear integro-differential evolution problems arising in option pricing: a viscosity solutions approach, Differential Integral Equations 16 (2003), no. 7, 787-811.

[6] A. L. Amadori, Quasi-variational inequalities with Dirichlet boundary condition related to exit time problems for impulse control, SIAM J. Control Optim. 43 (2004), no. 2, 570-589.

[7] A. L. Amadori, K. Karlsen, and C. La Chioma, Non-linear degenerate integro-partial differential evolution equations related to geometric Lévy processes and applications to backward stochastic differential equations, Stoch. Stoch. Rep. 76 (2004), no. 2, $147-177$. 
[8] D. Applebaum, Lévy processes-from probability to finance and quantum groups, Notices Amer. Math. Soc. 51 (2004), no. 11, 1336-1347.

[9] D. Applebaum, On the infinitesimal generators of Ornstein-Uhlenbeck processes with jumps in Hilbert space, Potential Anal. 26 (2007), no. 1, 79-100.

[10] D. Applebaum, Lévy processes and stochastic calculus, Second edition, Cambridge Studies in Advanced Mathematics, 116, Cambridge University Press, Cambridge, 2009 .

[11] M. Arisawa, A new definition of viscosity solutions for a class of second-order degenerate elliptic integro-differential equations, Ann. I. H. Poincaré 23 (2006), 695-711.

[12] G. Barles, R. Buckdahn, and E. Pardoux, Backward stochastic differential equations and integral-partial differential equations, Stochastics Stochastics Rep. 60 (1997), no. $1-2,57-83$.

[13] G. Barles, P. Cardaliaguet, O. Ley, and A. Monteillet, Uniqueness results for nonlocal Hamilton-Jacobi equations, J. Funct. Anal. 257 (2009), no. 5, 1261-1287.

[14] G. Barles, P. Cardaliaguet, O. Ley, and A. Monteillet, Existence of weak solutions for general nonlocal and nonlinear second-order parabolic equations, Nonlinear Anal. 71 (2009), no. 7-8, 2801-2810.

[15] G. Barles, E. Chasseigne, and C. Imbert, On the Dirichlet problem for second-order elliptic integro-differential equations, Indiana Univ. Math. J. 57 (2008), no. 1, 213246.

[16] G. Barles, E. Chasseigne, and C. Imbert, Hölder continuity of solutions of secondorder non-linear elliptic integro-differential equations, J. Eur. Math. Soc. (JEMS) 13 (2011), no. 1, 1-26.

[17] G. Barles, and C. Imbert, Second-order elliptic integro-differential equations: Viscosity solutions' theory revisited, Ann. Inst. H. Poincaré Anal. Non Linéaire 25 (2008), no. 3, 567-585.

[18] R. F. Bass, and M. Kassmann, Hlder continuity of harmonic functions with respect to operators of variable order, Comm. Partial Differential Equations 30 (2005), no. 7-9, 1249-1259.

[19] I. Bensaoud, and A. Sayah, Stability results for Hamilton-Jacobi equations with integro-differential terms and discontinuous Hamiltonians, Arch. Math. (Basel) 79 (2002), 392-395. 
[20] F. E. Benth,K. H. Karlsen, and K. Reikvam, Optimal portfolio selection with consumption and nonlinear integro-differential equations with gradient constraint: a viscosity solution approach, Finance Stoch. 5 (2001), no. 3, 275-303.

[21] F. E. Benth,K. H. Karlsen, and K. Reikvam, Portfolio optimization in a Lvy market with intertemporal substitution and transaction costs, Stoch. Stoch. Rep. 74 (2002), no. 3-4, 517-569.

[22] J. Bertoin, Lévy processes, Cambridge University Press, 1996.

[23] I. H. Biswas, E. R. Jakobsen, and K. H. Karlsen, Viscosity solutions for a system of integro-PDEs and connections to optimal switching and control of jump-diffusion processes, Appl. Math. Optim. 62 (2010), no. 1, 47-80.

[24] L. Caffarelli, and L. Silvestre, Regularity theory for fully nonlinear integro-differential equations, Comm. Pure Appl. Math. 62 (2009), no. 5, 597-638.

[25] E. Chasseigne, The Dirichlet problem for some non-local diffusion equations, Diff. Int. Eq. 20 (2007), 1389-1404.

[26] M. G. Crandall, H. Ishii, and P. L. Lions, User's Guide to viscosity solutions of second order partial differential equations, Bull. Amer. Math. Soc. 27 (1992), 1-67.

[27] M. G. Crandall, M. Kocan, and A. Święch, On partial sup-convolutions, a lemma of P.L. Lions and viscosity solutions in Hilbert spaces, Adv. Math. Sc. Appl. 3 (1993/4), 1-15.

[28] M. G. Crandall, and P. L. Lions, Viscosity solutions of Hamilton-Jacobi equations in infinite dimensions. IV. Hamiltonians with unbounded linear terms, J. Funct. Anal. 90 (1990), 237-283.

[29] M. G. Crandall, and P. L. Lions, Viscosity solutions of Hamilton-Jacobi equations in infinite dimensions. V. Unbounded linear terms and B-continuous solutions, J. Funct. Anal. 97 (1991), 417-465.

[30] G. Da Prato, and J. Zabczyk, Stochastic equations in infinite dimensions, Encyclopedia of Mathematics and its Applications, 44, Cambridge University Press, Cambridge, 1992 .

[31] I. Ekeland, and G. Lebourg, Generic Frechet-differentiability and perturbed optimization problems in Banach spaces, Trans. Amer. Math. Soc. 224 (1977), 193-216. 
[32] M. G. Garroni, and J. L. Menaldi, Green functions for second order parabolic integrodifferential problems, Pitman Research Notes in Mathematics Series, 275. Longman Scientific \& Technical, Harlow; copublished in the United States with John Wiley \& Sons, Inc., New York, 1992.

[33] M. G. Garroni, and J. L. Menaldi, Maximum principles for integro-differential parabolic operators, Differential Integral Equations 8 (1995), no. 1, 161-182.

[34] M. G. Garroni, and J. L. Menaldi, Second order elliptic integro-differential problems, Chapman \& Hall/CRC Research Notes in Mathematics, 430. Chapman \& Hall/CRC, Boca Raton, FL, 2002.

[35] F. Gimbert, and P. L. Lions, Existence and regularity results for solutions of secondorder, elliptic integro-differential operators, Ricerche Mat. 33 (1984), no. 2, 315-358.

[36] F. Gimbert, and P. L. Lions, On the regularity of solutions of the Dirichlet problem for elliptic integro-differential operators: a counterexample, Ricerche Mat. 34 (1985), no. 2, 283-288.

[37] C. Imbert, A non-local regularization of first order Hamilton-Jacobi equations, J. Differential Equations 211 (1) (2005) 218-246.

[38] H. Ishii, Viscosity solutions for a class of Hamilton-Jacobi equations in Hilbert spaces, J. Funct. Anal. 105 (1992), 301-341.

[39] Y. Ishikawa, Optimal control problem associated with jump processes, Appl. Math. Optim. 50 (2004), no. 1, 21-65.

[40] E. R. Jakobsen, and K. H. Karlsen, Continuous dependence estimates for viscosity solutions of integro-PDEs, J. Differential Equations 212 (2005), no. 2, 278-318.

[41] E. R. Jakobsen, and K. H. Karlsen, A "maximum principle for semicontinuous functions" applicable to integro-partial differential equations, NoDEA Nonlinear Differential Equations Appl. 13 (2006), 137-165.

[42] G. Kallianpur, and J. Xiong, Stochastic differential equations in infinite-dimensional spaces, Institute of Mathematical Statistics Lecture Notes-Monograph Series, 26, Institute of Mathematical Statistics, Hayward, CA, 1995.

[43] M. Kassmann, The theory of De Giorgi for non-local operators, C. R. Math. Acad. Sci. Paris 345 (2007), no. 11, 621-624. 
[44] M. Kassmann, A priori estimates for integro-differential operators with measurable kernels, Calc. Var. Partial Differential Equations 34 (2009), no. 1, 1-21.

[45] D. A. Kelome, Viscosity solutions of second order equations in a separable Hilbert space and applications to stochastic optimal control, Thesis (Ph.D.)Georgia Institute of Technology, 2002, 106 pp.

[46] D. Kelome, and A. Święch, Perron's method and the method of relaxed limits for "unbounded" PDE in Hilbert spaces Studia Math. 176 (2006), no. 3, 249-277.

[47] S. Lenhart, Integro-differential operators associated with diffusion processes with jumps, Appl. Math. Optim. 9 (1982/83), no. 2, 177-191.

[48] S. Lenhart, and Y. C. Liao, Integro-differential equations associated with optimal stopping time of a piecewise-deterministic process, Stochastics 15 (1985), no. 3, 183207.

[49] P. Lescot, and M. Röckner, Perturbations of generalized Mehler semigroups and applications to stochastic heat equations with Levy noise and singular drift, Potential Anal. 20 (2004), no. 4, 317-344.

[50] P. L. Lions, Viscosity solutions of fully nonlinear second-order equations and optimal stochastic control in infinite dimensions. Part III. Uniqueness of viscosity solutions of general second order equations, J. Funct. Anal. 86 (1989), 1-18.

[51] J. L. Menaldi, and M. Robin, On optimal ergodic control of diffusions with jumps, Stochastic analysis, control, optimization and applications, 439-456, Systems Control Found. Appl., Birkhuser Boston, Boston, MA, 1999.

[52] R. Mikulyavichyus, and G. Pragarauskas, On Hlder continuity of solutions of certain integro-differential equations, Ann. Acad. Sci. Fenn. Ser. A I Math. 13 (1988), no. 2, $231-238$.

[53] R. Mikulyavichyus, and G. Pragarauskas, On the uniqueness of a solution to the Bellman equation in Sobolev's classes, Litovsk. Mat. Sb. 31 (1991), no. 4, 646-664; translation in Lithuanian Math. J. 31 (1991), no. 4, 449-464 (1992).

[54] R. Mikulyavichyus, and G. Pragarauskas, On the Cauchy problem for certain integrodifferential operators in Sobolev and Hlder spaces, Liet. Mat. Rink. 32 (1992), no. 2, 299-331; translation in Lithuanian Math. J. 32 (1992), no. 2, 238-264 (1993). 
[55] R. Mikulyavichyus, and G. Pragarauskas, On classical solutions of certain nonlinear integro-differential equations, Stochastic processes and optimal control (Friedrichroda, 1992), 151-163, Stochastics Monogr., 7, Gordon and Breach, Montreux, 1993.

[56] R. Mikulyavichyus, and G. Pragarauskas, Classical solutions of boundary value problems for some nonlinear integro-differential equations, (Russian) Liet. Mat. Rink. 34 (1994), no. 3, 347-361; translation in Lithuanian Math. J. 34 (1994), no. 3, 275-287 (1995).

[57] R. Mikulyavichyus, and G. Pragarauskas, Nonlinear potentials of the CauchyDirichlet problem for the Bellman integro-differential equation, (Russian) Liet. Mat. Rink. 36 (1996), no. 2, 178-218; translation inLithuanian Math. J. 36 (1996), no. 2, 142-173 (1997).

[58] R. Mikulyavichyus, and G. Pragarauskas, On Cauchy-Dirichlet problem for linear integro-differential equation in weighted Sobolev spaces, Stochastic differential equations: theory and applications, 357-374, Interdiscip. Math. Sci., 2, World Sci. Publ., Hackensack, NJ, 2007.

[59] B. Oksendal, and A. Sulem, Applied stochastic control of jump diffusions, Second edition, Universitext, Springer, Berlin, 2007.

[60] S. Peszat, Lévy-Ornstein-Uhlenbeck transition semigroup as second quantized operator, J. Funct. Anal. 260 (2011), no. 12, 3457-3473.

[61] S. Peszat, and J. Zabczyk, Stochastic partial differential equations with Lévy noise. An evolution equation approach, Encyclopedia of Mathematics and its Applications, 113, Cambridge University Press, Cambridge, 2007.

[62] H. Pham, Optimal stopping of controlled jump diffusion processes: a viscosity solution approach, J. Math. Systems Estim. Control 8 (1998), no. 1, 27 pp.

[63] E. Priola, Pathwise uniqueness for singular SDE's driven by stable processes, to appear in Osaka J. Math..

[64] E. Priola, and J. Zabczyk, Liouville theorems for non-local operators, J. Funct. Anal. 216 (2004), no. 2, 455-490.

[65] E. Priola, and J. Zabczyk, Structural properties of semilinear SPDE's driven by cylindrical stable processes, Probab. Theory Related Fields 149 (2011), 97-137. 
[66] M. Renardy, Polar decomposition of positive operators and a problem of Crandall and Lions, Appl. Anal. 57 (1995), no. 3-4, 383-385.

[67] K. Sato, Lévy processes and infinitely divisible distributions, Cambridge University Press, 1999.

[68] A. Sayah, Équations d'Hamilton-Jacobi du premier ordre avec termes intgrodiffrentiels. I. Unicité des solutions de viscosité. II. Existence de solutions de viscosité, Comm. Partial Differential Equations 16 (6-7) (1991) 1057-1093.

[69] L. Silvestre, Hölder estimates for solutions of integro-differential equations like the fractional Laplace, Indiana Univ. Math. J. 55 (2006), no. 3, 1155-1174.

[70] L. Silvestre, On the differentiability of the solution to the Hamilton-Jacobi equation with critical fractional diffusion, Adv. Math. 226 (2011), no. 2, 2020-2039.

[71] H. M. Soner, Optimal control with state-space constraint. II, SIAM J. Control Optim. 24 (6) (1986) 1110-1122.

[72] H. M. Soner, Optimal control of jump-Markov processes and viscosity solutions, in Stochastic differential systems, stochastic control theory and applications (Minneapolis, Minn., 1986), 501-511, IMA Vol. Math. Appl., 10, Springer, New York, 1988.

[73] A. Święch, A PDE approach to large deviations in Hilbert spaces, Stochastic Process. Appl. 119 (2009), no. 4, 1081-1123.

[74] A. Święch, and J. Zabczyk, Large deviations for stochastic PDE with Lévy noise, J. Funct. Anal. 260 (2011), 674-723.

[75] J. Zabczyk, Bellman's inclusions and excessive measures, Probab. Math. Statist. 21 (2001), no. 1, 101-122. 